\title{
Coordination with erroneous communication: results of an agent-based simulation
}

\author{
Friederike Wall ${ }^{1}$
}

Received: 5 January 2018 / Revised: 5 October 2018 / Accepted: 24 November 2018 /

Published online: 14 December 2018

(c) The Author(s) 2018

\begin{abstract}
Coordination among agents performing interdependent tasks in a distributed problem-solving system is of crucial relevance with respect to the overall performance achieved by the system. Various forms of coordination require communication among agents which may accidentally be distorted. This paper investigates how sensitive different coordination mechanisms, employed in distributed problem-solving systems, are to unintentional communication errors. For this, an agent-based simulation based on the framework of NK fitness landscapes is employed. The study controls for different levels of erroneous communication and complexity of the problems to be solved collaboratively. The results provide broad, though not universal support for detrimental effects of erroneous communication and suggest that the various coordination mechanisms under investigation show different sensitivity to communication errors. Moreover, the results indicate that the complexity of the overall problem to be solved and the objectives pursued by the agents considerably shape the robustness of coordination mechanisms against erroneous communication. The results shed some new light on the relation between communication and coordination and, in particular, may contribute to the organizational design of error tolerant systems.
\end{abstract}

Keywords Agent-based simulation · Communication errors · Complexity · Coordination · Multiple controls $\cdot$ NK fitness landscapes $\cdot$ Robustness

\section{Introduction}

When interdependent tasks are assigned to different agents, coordination becomes relevant in order to avoid losses with respect to the overall performance achieved by the system $[32,49]$. The coordination of autonomous agents' actions is an interdisciplinary field of research employing ideas from various disciplines like organization theory, economics or computer science to name but a few (e.g., $[6,8,9,37,52,60])$. Dependent on the context, different ways to coordinate agents' actions may be employed—eventually in combination [26]: among the

Friederike Wall

friederike.wall@aau.at

1 Department of Management Control and Strategic Management, Alpen-Adria-Universität Klagenfurt, Universitätsstraße 65-67, Klagenfurt, Austria 
ways of coordination are, for example, the sharing of common values, incentives provided to the agents or rules of how agents have to seek for consent and adjustment with other agents (e.g., [37,46]). Particularly the latter mechanisms of coordination may employ direct communication among the agents, i.e., agents informing each other about the intended actions and, eventually, negotiating and mutually adjusting their plans (plan revision) [19,51,52].

It is well recognized that, in the course of coordination, communication poses several problems: communication may be costly and, thus, agents may have an incentive to avoid communication or agents may deliberately distort communication in order to obtain an advantage $[16,19]$. While related in spirit, the research effort introduced in this paper addresses a different aspect of imperfect communication for coordination: unintentional communication errors may occur when the sender accidentally distorts some components of its message or when the transmission does not work correctly.

Against this background the paper seeks to contribute to two interrelated research questions: First, how sensitive are mechanisms for coordinating plans of autonomous agents against erroneous communication in the context of different levels of coordination need? Second, are the coordination mechanisms less sensitive against communication errors when they are combined with other coordination practices? The latter aspect, in particular, could provide some insights into the design of robust coordination systems in the sense that multiple components for coordination are combined (so-called "multiple controls") with taking the interrelations into account $[5,8,26]$. This paper is an extended version of [58] which was presented at the 14th International Conference on Distributed Computing and Artificial Intelligence (DCAI). The extensions are as follows: (1) an additional mechanism of coordination is studied which is of particular interest since it captures the involvement of a central decision-making agent; (2) the study is deepened in terms of an in-depth analysis of effects of communication errors in the different coordination mechanisms; (3) the sensitivity of coordination mechanisms to communication errors is additionally studied in the context of different types of agents' objective functions capturing different incentives provided.

For this, the study uses an agent-based simulation and, in particular, the structure of NK fitness landscapes is applied which was originally introduced in the domain of evolutionary biology [27,28] and, since then, broadly employed (for an overview see [55]). This methodological approach was chosen for the following reasons: first, simulation appears an appropriate approach to map and systematically variegate errors in different organizational settings as captured, for example, by the coordination mechanisms employed [15]; second, an agent-based model allows to explicitly depict heterogeneous and locally acting agents and their interactions based on - potentially imperfect—communication; third, a major advantage of NK landscapes is that they allow to systematically vary the complexity of a task in terms of the interdependencies among the sub-tasks [35].

The remainder of this paper is organized as follows: Sect. 2 places the research questions of this paper into the context of related work on imperfect communication. Section 3 describes the simulation model before, in Sect. 4, the different types of simulation experiments, and the related parameter settings are introduced. In Sect. 5, the results of the simulation experiments are presented and discussed and Sect. 6 provides some concluding remarks and directs to future research. 


\section{Related work}

Coordination and imperfect communication are fundamental issues whenever autonomous agents collaborate. Accordingly, the scientific domains addressing this subject are manifold such as, for example, economics (in particular, information economics and game theory) and multi-agent systems (in particular, multi-agent planning). Regarding the latter, it is worth mentioning that this study is mainly related to what is called "plan merging," i.e., modes of integrating partial plans into an overall plan (e.g., $[18,25,60])$. For relating this study to prior research, it appears helpful to build on the "types" of imperfections in the communication for coordination. For this, at least, two dimensions of imperfect communication may be distinguished: (1) time delays in communication and (2) content-related errors of communication.

Time delays are particularly relevant in dynamical systems where agents have to exchange information about their current states (e.g., position, velocity) to (some or all) other agents in order to enable these agents to employ these information in their further actions. Delays which could considerably reduce the performance of the system, may, for example, result from limited communication speed or additional time required to compute certain control inputs [8]. In the domain of multi-agent systems, the periodic transmission which requires synchronization among the agents and the (newer) event-driven consensus are employed $[2,23,24,40]$. It has been shown that even the loss of information (packets) in the course of communication for coordination can be regarded as a special case of coordination with delayed communication [8].

With respect to research on content-related imperfections of communication in the context of coordination, the differentiation between intentional and unintentional imperfections [30] appears relevant—particularly because even the underlying assumptions about agents may differ remarkably:

In the domains of information economics and game theory, theoretical and experimental studies show that even the communication of non-verifiable and non-binding information ("cheap talk") has positive effects on coordination-presumably because it stabilizes the mutual expectations on the opponents' behavior (e.g., [10,11,19,21]). Another strand of research with long tradition, particularly, in the branches of contract theory and game theory, studies the effects of deliberately "shaped"-for not to say: manipulated-information in the context of coordination. The key idea is that self-interested agents purposefully may shape the information they communicate in order to achieve certain advantages over the other parties. A prerequisite is that information asymmetries among parties exist which cannot be resolved - at least not at reasonable costs; moreover, parties are assumed to be inclined to exploit these asymmetries for their own best interests and being capable to anticipate the effects of their communication on the behavior of the other parties (for overviews, e.g., $[29,33,36])$. In this vein, considerable analytical and experimental research was directed towards the coordination by incentive schemes which induce truth-telling behavior of agents (for overviews [13,42]). For example, for coordination by budgets a prominent idea is to employ a compensation function with a component that over-proportionally increases with the ex-ante reported numbers plus a component capturing the deviation of the ex-post (actual) from the ex-ante numbers whose gradient is given by the level of the ex-ante report ([38], for a recent overview [14]). Coordination mechanisms based on ideas of information economics and game theory have found high interest also in the domain of multi-agent systems (e.g., $[3,22,39])$. 
However, this stream of research has been challenged for some aspects: first, it usually studies communication for coordination in rather "reduced" settings often comprising just two agents and with rather limited information flows required for coordination. Therefore, it has been questioned in how far the results can be transferred to more realistic settings comprising more agents where, additionally, communication is related to a certain task to be accomplished and where information flows are more complex [53].

Second, research in the vein of information economics and game theory builds on some fundamental assumptions about agents as, for example, agents being capable to overlook their entire solution space at once and, therefore, "instantaneously" maximizing their individual utility functions while perfectly anticipating other parties' behavior. These assumptions were challenged, at least, dating back to the seminal works of Simon $[47,48]$ according to which satisficing and stepwise search (rather than optimization) under imperfect information are more appropriate and realistic assumptions for many contexts. Simon's ideas about agents were of considerable influence, not only in managerial science (and, particularly, in the vein of social simulation, e.g., [55]) but also in the domain of multi-agent systems [39,50].

Hence, with respect to content-related imperfections of communication for coordination, the aforementioned issues suggest a different perspective than taken in the "traditional" view inspired by information economics and game theory: settings of several agents endowed with limited information-processing capabilities which exchange information according to certain coordination mechanisms to collaboratively perform a task; in the course of coordination, communication may be subject to imperfect anticipations of the exchanged information's effects on other agents choices and affected by unintentional failures on the sender's site or from "accidentally" occurring obstacles in the communication channels.

With this, the content-related imperfections studied in this paper are of an unintentional nature in the meaning of being "accidental" or erroneous. Moreover, the research endeavor of this study does not address imperfections in communication due to time delays (see above); rather, in the model, it is assumed that periodically, i.e., in the end of each time step, and without any failures in synchronization agents communicate their individual plans as determined in the particular coordination mechanism - though, eventually, exchanging erroneously distorted contents.

\section{Simulation model}

In the simulation model, distributed problem-solving systems (DPSS)—comprising distributed search agents and some organizational arrangements for task assignment and coordination - collaboratively search for superior solutions to an overall problem to be solved. The principle processual structure of the simulation model is depicted in Fig. 1, and, subsequently, the model is described into detail.

\subsection{Distributed problem and task assignment}

The problem-solving systems simulated face an $N$-dimensional binary search problem according to the framework of NK fitness landscapes. From a more "technical" point of view, NK landscapes are stochastically generated pseudo-boolean functions with $N$ bits, i.e., $F:\{0,1\}^{N} \rightarrow \mathbb{R}^{+}[1,35]$. The complexity of the $N$-dimensional search problem, in terms of interdependencies among the $N$ bits (choices), is captured by parameter $K$. 


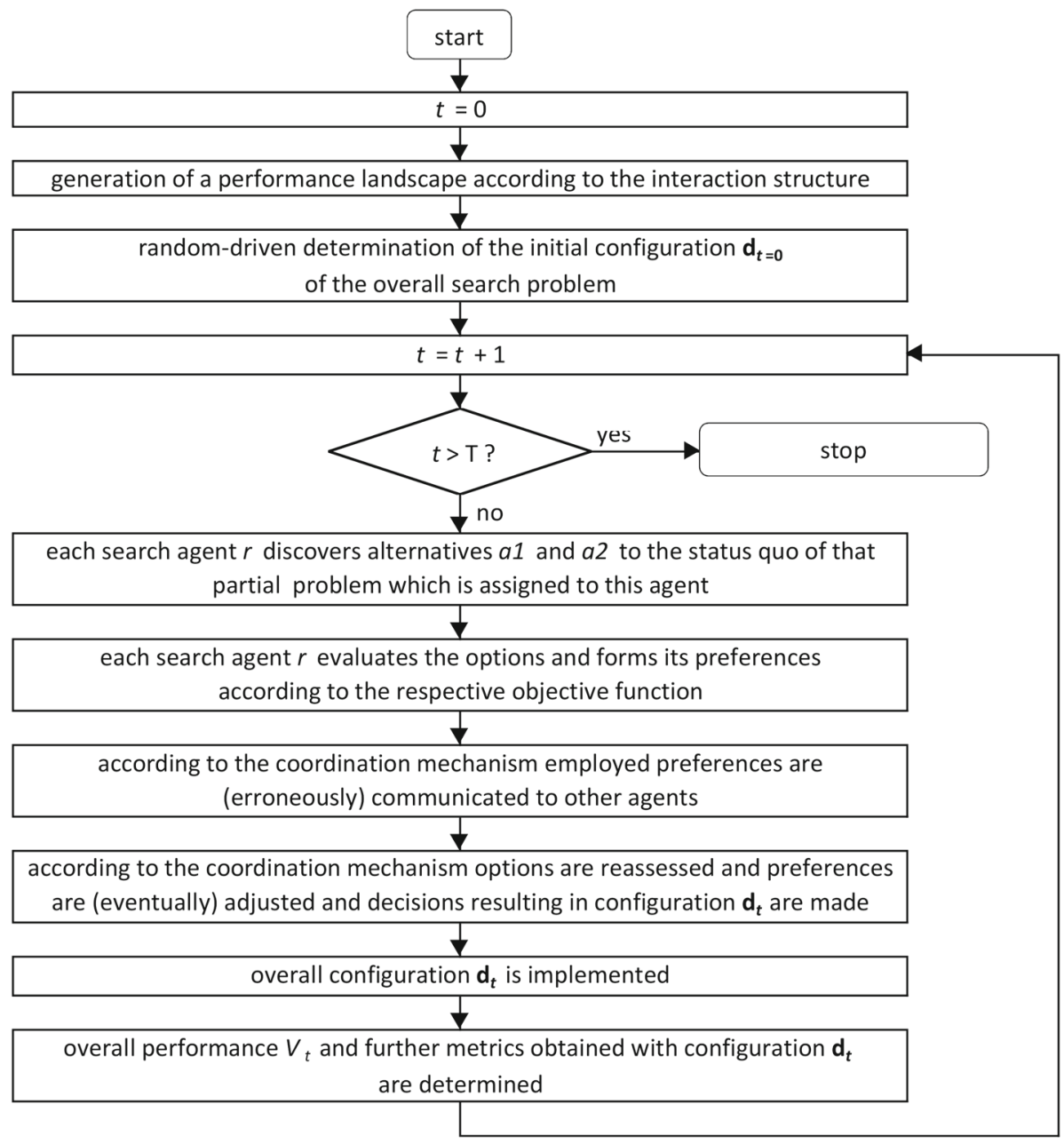

Fig. 1 Principle processual structure of the simulation model

In particular, in each time step, a system of distributed search agents seeks for a superior configuration $\mathbf{d}_{\mathbf{t}}$ of an $N$-dimensional binary problem, i.e., $\mathbf{d}_{\mathbf{t}}=\left(d_{1 t}, \ldots, d_{N t}\right)$ with $d_{i t} \in$ $\{0,1\}, i=1, \ldots, N$, out of $2^{N}$ different binary vectors possible. Each of the two states $d_{i t} \in\{0,1\}$ contributes with $C_{i t}$ to overall system's performance $V\left(\mathbf{d}_{\mathbf{t}}\right)$. In line with the NK framework, $C_{i t}$ is randomly drawn from a uniform distribution with $0 \leq C_{i t} \leq 1$. The parameter $K$ (with $0 \leq K \leq N-1$ ) reflects the number of those choices $d_{j t}, j \neq i$ which also affect the performance contribution $C_{i t}$ of choice $d_{i t}$. Hence, contribution $C_{i t}$ may not only depend on the single choice $d_{i t}$ but also on $K$ other choices:

$$
C_{i t}=f_{i}\left(d_{i t} ; d_{i_{1} t}, \ldots d_{i_{K} t}\right) \text {, }
$$

with $\left\{i_{1}, \ldots, i_{K}\right\} \subset\{1, \ldots, i-1, i+1, \ldots, N\}$.

In case of no interactions among choices, $K$ equals 0 , and $K$ is $N-1$ for maximum interactions where each single choice $d_{i t}$ affects the performance contribution of each other binary 
choice $d_{j t}, j \neq i$. The overall performance $V_{t}$ achieved in period $t$ results as normalized sum of contributions $C_{i t}$ from

$$
V_{t}=V\left(\mathbf{d}_{\mathbf{t}}\right)=\frac{1}{N} \sum_{i=1}^{N} C_{i t}
$$

The distributed systems simulated consist of $M$ agents indexed by $r=1, \ldots, M$. The agents collaboratively search for superior solutions of the $N$-dimensional binary search problem $\mathbf{d}_{\mathbf{t}}$ which is partitioned into $M$ disjoint $N^{r}$-dimensional subproblems of equal size with $N^{*}=N / M \forall r$ binary choices. Each subproblem is exclusively assigned to one agent $r$. Hence, in each time step $t$, search agent $r$ has primary control over a subset of $N^{*}$ single choices of the $N$-dimensional problem. With this, the overall search problem $\mathbf{d}_{\mathbf{t}}=\left(d_{1 t}, \ldots, d_{N t}\right)$ can also be expressed by the combination of partial problems as $\mathbf{d}_{\mathbf{t}}=\left[\mathbf{d}_{t}^{1}, \ldots, \mathbf{d}_{t}^{r}, \ldots, \mathbf{d}^{M_{t}}\right]$ with each agent's choices related only to its own partial decision problem $\mathbf{d}_{t}^{r}$. In every time step $t$ of the search, each agent makes a plan regarding its partition of the overall problem which is described in Sect. 3.2. Then, the coordination mode employed determines to which other agents $q \neq r$ an agent $r$ communicates its plan and how the plans may be aligned (Sects. 3.3 and 3.4).

\subsection{Search agents' preferences}

In each time step $t$, an agent seeks to identify the best configuration for the "own" choices $\mathbf{d}_{\mathbf{t}}^{\mathbf{r}}$. However, as motivated in Sect. 2, this study assumes that the agents are endowed with rather limited information-processing capabilities. In the model, this is reflected in several ways, i.e.,

1. in a rather limited number of new options that the agents discover: each agent discovers two new configurations;

2. in agents' limited memory of options that have been chosen in previous periods: agents only remember the option $\mathbf{d}_{\mathbf{t}-\mathbf{1}}^{\mathbf{r} *}$ implemented in the previous period, i.e., the status quo and

3. limited anticipation of the fellow agents' actions, i.e., agent $r$ assumes that the other agents $q \neq r$ stay with their choices of the previous period.

In particular, in each time step $t$, agent $r$ randomly discovers two alternatives to the status quo $\mathbf{d}_{\mathbf{t}-\mathbf{1}}^{\mathbf{r} *}$-one that differs in one choice $(a 1)$ and another $(a 2)$ where two choices are modified compared to the status quo. Hence, in time step $t$, agent $r$ has three options to choose from: to keep the status quo or to switch to $\mathbf{d}_{\mathbf{t}}^{\mathbf{r}, \mathbf{a} 1}$ or $\mathbf{d}_{\mathbf{t}}^{\mathbf{r}, \mathbf{a} 2}$.

For the formation of preferences at time step $t$, an agent $r$ assesses these options according to their effects on the agent's objective. In many contexts, the agents' objective functions are subject to the system's design. For example, think of the incentives provided to managers of a firm: the unit managers may be rewarded according to their particular units' performance or according to the performance of the entire firm. In the latter case, the managers have an incentive to take the effects of their choices on the overall performance into account. Hence, the appropriate design of the objective function (via incentives) represents a way to coordinate the choices of distributed search agents [56].

The model differentiates between two possible types of objective functions for the search agents, i.e., two possible levels of coordination via (alignment of) objectives are captured in the model:

Parochial type Each search agent $r=1, \ldots, M$ seeks to find a superior solution for its "own" partial decision problem - or in other words: the objective of search agent $r$ is to maximize 
the performance resulting from the solution to the particular subproblem assigned to that agent:

$$
P_{t}^{r}\left(\mathbf{d}_{t}^{r}\right)=\frac{1}{N} \sum_{i=1+(r-1) N^{*}}^{r N^{*}} C_{i t}
$$

where $N^{*}=N^{r}=N / M \forall r$. With this, agent $r$ focuses on the performance of the partial vector $\mathbf{d}_{t}^{r}$ which is in its own primary control. In each time step, search agent $r$ decides in favor of that option out of the three alternatives $d_{t-1}^{r *}, d_{t}^{r}, \mathbf{a 1}$ or $d_{t}^{r, a 2}$ which promises the highest performance $P_{t}^{r}$.

Overall type In every time step $t$, each search agent $r$ seeks to find that solution out of the three options related to its partial problem which results in the highest overall performance of the system. Hence, in this case, Eq. 2 denotes the objective function of each single search agent.

In every time step $t$, each search agent evaluates its options according to the respective objective function given and, based on the evaluation, compiles a list of preferences $L_{t}^{r}=\left\{\mathbf{d}_{\mathbf{t}}^{\mathbf{r}, \mathbf{p} 1}, \mathbf{d}_{\mathbf{t}}^{\mathbf{r}, \mathbf{p} \mathbf{2}}, \mathbf{d}_{\mathbf{t}}^{\mathbf{r}, \mathbf{p} 3}\right\} \cdot \mathbf{d}_{\mathbf{t}}^{\mathbf{r}, \mathbf{p} \mathbf{1}}$ indicates the most preferred option according to the agent's objective; correspondingly, $\mathbf{d}_{\mathbf{t}}^{\mathbf{r}, \mathbf{p} 2}$ denotes the second-most and $\mathbf{d}_{\mathbf{t}}^{\mathbf{r}, \mathbf{p} 3}$ the least preferred option out of the three. Hence, as a result of the agents' search and these first assessments of options, each agent $r$ has an ordered list $L_{t}^{r}$ of preferences which we call agent $r$ 's plan.

It is worth mentioning that, so far, the preferences are formed without knowing what the other agents intend to opt for; rather, this first formation of preferences is based on the assumption that the other agents $q \neq r$ stay with the status quo, i.e., those partial configurations that were implemented in the previous period. Subject to the coordination mechanism as introduced in Sect. 3.4, the other agents' intentions are considered and, eventually, reflected in revised plans. However, this requires that the agents' intentions as captured in $L_{t}^{r}$ are communicated within the search system.

\subsection{Erroneous communication}

In the very center of this study is erroneous communication occurring in the course of coordination - may it be due to accidental faults of sending on the senders' site or imperfect transmission of plans. However, for the sake of simplicity, in this study it is assumed that the erroneously communicated options of a plan are the same for all the recipients of that plan (which is particular reasonable if communicative faults occur on the sender's site). The level of erroneous communication is captured by the number $E^{C}$ of single choices $i$ out of the $N$-dimensional search problem which are not communicated correctly in the course of coordination.

In particular, after the agents have made their plans for period $t$ as captured in $L_{t}^{r} \forall r \in$ $\{1, \ldots, M\}$ and before they inform each other about their plans, a list $Q_{t}$ with $\left|\left(Q_{t}\right)\right|=E^{C}$ entries is generated randomly. The list $Q_{t}$ contains those single choices (bits) $i$ out of the $N$ dimensions of the binary search problem which will be flipped in the communication among agents in time step $t$. For example, if the error level is $E^{C}=3$ and $Q_{t}=\{2,6,12\}$ the second, the sixth and the twelfth bit will be flipped when it comes to communication of the agents' plans. (Recall that the choice of each $i$ is unambiguously assigned to one of the $M$ agents.) Each of the $N$ single choices has the same probability to be chosen for the list $Q_{t}$.

Hence, before it comes, eventually, to the revision of plans, the "true" plans $L_{t}^{r}$ are distorted according to $Q_{t}$. The resulting distorted plan $\widetilde{L}_{t}^{r}$ is a function of the "true" plans and the list 
of distortions $Q_{t}$ :

$$
\widetilde{L}_{t}^{r}=\widetilde{L}_{t}^{r}\left(L_{t}^{r}, Q_{t}\right)
$$

with $\widetilde{L}_{t}^{r}$ capturing “distorted" options $\widetilde{L}_{t}^{r}=\left\{\tilde{\mathbf{d}}_{\mathbf{t}}^{\mathbf{r}, \mathbf{p} 1}, \tilde{\mathbf{d}}_{\mathbf{t}}^{\mathbf{r}, \mathbf{p} 2}, \tilde{\mathbf{d}}_{\mathbf{t}}^{\mathbf{r}, \mathbf{p} 3}\right\}$.

\subsection{Coordination mechanisms}

Apart from, eventually, letting the search agents pursue the objective of overall performance rather than their parochial performances (see Sect. 3.2), the simulation model captures three mechanisms to coordinate the partial plans:

Sequential mode The sequential mode captures what is also named "sequential planning." The search agents make their choices sequentially where, for the sake of simplicity, the sequence is given by the index $r$ of the agents. In particular, in time step $t$ agent $r$ with $2 \leq r \leq M$ is, eventually imperfectly, informed by agent $r-1$ about the choices made so far, i.e., made by the "preceding" agents $<r$. Agent $r$ takes these choices into account and re-evaluates its "true" own options $\mathbf{d}_{\mathbf{t}-\mathbf{1}}^{\mathbf{r} *}, \mathbf{d}_{\mathbf{t}}^{\mathbf{r}, \mathbf{a} 1}$ and $\mathbf{d}_{\mathbf{t}}^{\mathbf{r}, \mathbf{a} 2}$ according to its objective function, i.e., Eqs. 3 or 2, respectively. This may potentially result in an adjusted list of preferences $L_{t}^{r *}=\left\{\mathbf{d}_{\mathbf{t}}^{\mathbf{r}, \mathbf{p} \mathbf{1}}, \mathbf{d}_{\mathbf{t}}^{\mathbf{r}, \mathbf{p} \mathbf{2}}, \mathbf{d}_{\mathbf{t}}^{\mathbf{r}, \mathbf{p} \mathbf{3}}\right\}$ from which (the eventually "new") $\mathbf{d}_{\mathbf{t}}^{\mathbf{r}, \mathbf{p 1}}$ is chosen. $\mathbf{d}_{\mathbf{t}}^{\mathbf{r}, \mathbf{p} \mathbf{1}}$ here depends on $\tilde{\mathbf{d}}_{\mathbf{t}}^{\mathbf{r}-\mathbf{1}, \mathbf{p} \mathbf{1}}$, i.e., is a nested function of the choices of the preceding agents and distorted according to $Q_{t}$. Only agent 1 does not have to take a previous choice into account. Hence, the choice of agent $r$ is made according to

$$
\mathbf{d}_{\mathbf{t}}^{\mathbf{r} *}= \begin{cases}\mathbf{d}_{\mathbf{t}}^{\mathbf{r}, \mathbf{p} 1}\left(\tilde{\mathbf{d}}_{\mathbf{t}}^{\mathbf{r}-1, \mathbf{p} 1}\right) & \text { for } 2 \leq r \leq M \\ \mathbf{d}_{\mathbf{t}}^{\mathbf{r}, \mathbf{p} 1} & \text { for } r=1\end{cases}
$$

and with Eq. (5) the overall configuration results from $\mathbf{d}_{\mathbf{t}}=\mathbf{d}_{\mathbf{t}}^{\mathbf{M} *}$.

Horizontal veto mode In the horizontal veto mode, first, the search agents mutually-and potentially erroneously_inform each other about their most preferred options $\tilde{\mathbf{d}}_{\mathbf{t}}^{\mathbf{r}, \mathbf{p} \mathbf{1}}$. Next, each agent $r$ evaluates the composite vector $\mathbf{d}^{\mathbf{r C}}$ according to Eqs. 3 or 2, respectively. $\mathbf{d}^{\mathbf{r C}}$ consists of the-potentially distorted-first preferences of the other agents $q \neq r$ and the "true" own preference, i.e., $\mathbf{d}^{\mathbf{r C}}=\left(\tilde{\mathbf{d}}_{\mathbf{t}}^{\mathbf{1}, \mathbf{p} \mathbf{1}}, \ldots, \mathbf{d}_{\mathbf{t}}^{\mathbf{r}, \mathbf{p} \mathbf{1}}, \ldots, \tilde{\mathbf{d}}_{\mathbf{t}}^{\mathbf{M}, \mathbf{p} \mathbf{1}}\right)$. However, the agents are endowed with mutual veto power: since no agent accepts a worse result than achieved in the previous period, the composite vector $\mathbf{d}^{\mathbf{r C}}$ requires to, at least, promise the same result with respect to each single agent's $r$ objective (see Sect. 3.2) as obtained with the status quo. Hence, if the search agents pursue a parochial objective according to Eq. 3, the following condition has to be fulfilled:

$$
P_{t}^{r}\left(\mathbf{d}^{\mathbf{r C}}\right) \geq P_{t}^{r}\left(\mathbf{d}_{\mathbf{t}-1}\right) \quad \forall r \in\{1, \ldots M\}
$$

In case that the search agents seek to increase the overall performance (Eq. 2), the following condition has to be met:

$$
V_{t}\left(\mathbf{d}^{\mathbf{r C}}\right) \geq V_{t}\left(\mathbf{d}_{\mathbf{t}-\mathbf{1}}\right) \quad \forall r \in\{1, \ldots M\}
$$

If condition 6 or 7 , respectively, is met for each agent $r$, the first "true" preferences $\mathbf{d}_{\mathbf{t}}^{\mathbf{r}, \mathbf{p} 1}$ of each agent are implemented. In contrast, if, at least, one agent vetoes, then the agents' second preferences - eventually distorted according to $Q_{t}$ - are composed by each agent to $\mathbf{d}^{\mathbf{r C}}=\left(\tilde{\mathbf{d}}_{\mathbf{t}}^{\mathbf{1}, \mathbf{p} \mathbf{2}}, \ldots \mathbf{d}_{\mathbf{t}}^{\mathbf{r}, \mathbf{p} \mathbf{2}}, \ldots \tilde{\mathbf{d}}_{\mathbf{t}}^{\mathbf{M}, \mathbf{p} \mathbf{2}}\right)$, evaluated according to Eq. 3 (or Eq. 2) and assessed in 
regard of Eq. 6 (or 7, respectively). If at least one agent vetoes (again, since the condition in Eqs. 6 or 7 is not met), then the status quo is kept, i.e., then $\mathbf{d}_{\mathbf{t}}=\mathbf{d}_{\mathbf{t}-\mathbf{1}}$; otherwise, the "true" second preferences $\mathbf{d}_{\mathbf{t}}^{\mathbf{r}, \mathbf{p} 2}$ are implemented.

Proposal mode In the proposal mode, a further agent comes into play, subsequently called the "central agent." This agent does not itself search for options but is merely in charge of deciding on the-eventually erroneously communicated-plans of the search agents (subject to the context, this central agent may, for example, be the headquarters of a firm or a central control unit for a fleet of unmanned aerial vehicles). The central agent seeks to increase the overall performance of the system according to Eq. 2.

In particular, in every period $t$, each search agent $r$ transfers its ordered list of preferences $L_{t}^{r}$ - eventually distorted to $\widetilde{L}_{t}^{r}$ - to the central agent. From these lists, the central agent compiles the first preferences to a composite vector $\mathbf{d}^{C}=\left(\tilde{\mathbf{d}}_{t}^{1, p 1}, \ldots \tilde{\mathbf{d}}_{t}^{r, p 1}, \ldots \tilde{\mathbf{d}}_{t}^{M, p 1}\right)$ and assesses the overall performance it promises according to Eq. 2. The central agent decides in favor of the composite vector $\mathbf{d}^{C}$ if it promises the same or a higher performance as the status quo $\mathbf{d}_{t-1}$ :

$$
V\left(\mathbf{d}^{C}\right) \geq V\left(\mathbf{d}_{t-1}\right)
$$

If the composite vector assembled from the search agents' (distorted) first preferences does not meet the condition in Eq. 8, then the central agent evaluates the vector composed from the search agents' - eventually distorted—second preferences according to Eq. 8. If this also does not, at least, promise the performance of the status quo, then the status quo is kept, i.e., then $\mathbf{d}_{t}=\mathbf{d}_{t-1}$. After the central agent has made its decision, it gives a signal to the search agents indicating whether the first or the second preference or even the status quo is to be implemented.

\section{Simulation experiments and parameter settings}

The experiments of this simulation study are intended to provide some insights on the effects of erroneous communication occurring in the course of the coordination of agents' plans in distributed problem-solving systems (DPSS). Therefore, the experiments distinguish for different levels of communication errors $E^{C}$ and for different levels of coordination need as shaped by the complexity of the underlying search problem. The processual structure of the experiments is depicted in Fig. 1. This section introduces the experiments and the related parameters settings (see Table 1).

In the simulation experiments, DPSS are observed for 250 periods ${ }^{1}$ while searching - with a start from a randomly chosen initial position in a performance landscape-for superior solutions with respect to overall performance $V_{t}$ (Eq. 2) to an $N=12$-dimensional binary search problem.

In the DPSS, the overall search problem is decomposed into $M$ subproblems of equal size, where each subproblem is exclusively delegated to one search agent. The vast majority of simulation experiments is conducted for $M=4$ agents; hence, if not indicated otherwise, the DPSS are assumed to comprise four agents. However, in order to illustrate the effects of communication errors in interrelation with the level of decomposition as given by the number

1 Pretests indicated that the key results of the simulations remain stable even for an observation period of $T=500$. 
Table 1 Parameter settings in the simulation experiments

\begin{tabular}{|c|c|c|c|c|}
\hline \multirow[t]{2}{*}{ Parameter } & \multirow[t]{2}{*}{ Values/types } & \multicolumn{3}{|c|}{ Experiments } \\
\hline & & $\mathrm{I}$ & II & III \\
\hline Observation period & $T=250$ & $\mathrm{x}$ & $\mathrm{x}$ & $\mathrm{x}$ \\
\hline Number of choices & $N=12$ & $\mathrm{x}$ & $\mathrm{x}$ & $\mathrm{x}$ \\
\hline \multirow[t]{4}{*}{ Interaction structures } & Block-diagonal: $K=2 ; K^{*}=0$ & - & $\mathrm{x}$ & $\mathrm{x}$ \\
\hline & Intermediate low: $K=3 ; K^{*}=1$; & $\mathrm{X}$ & $\mathrm{x}$ & $\mathrm{x}$ \\
\hline & Intermediate medium: $K=7 ; K^{*}=5$; & - & $\mathrm{x}$ & $\mathrm{x}$ \\
\hline & Full interdependent: $K=11 ; K^{*}=9$ & - & $\mathrm{x}$ & $\mathrm{x}$ \\
\hline \multirow[t]{4}{*}{ Number of search agents } & $M=4$ with agent $1: \mathbf{d}^{\mathbf{1}}=\left(d_{1}, d_{2}, d_{3}\right)$, & $\mathrm{x}$ & $\mathrm{x}$ & $\mathrm{x}$ \\
\hline & Agent 2: $\mathbf{d}^{2}=\left(d_{4}, d_{5}, d_{6}\right)$, agent $3: \mathbf{d}^{3}=$ & & & \\
\hline & $\left(d_{7}, d_{8}, d_{9}\right)$, agent $4: \mathbf{d}^{4}=\left(d_{10}, d_{11}, d_{12}\right)$ & & & \\
\hline & $M \in\{2,3,6\}$ & - & $\mathrm{x}$ & - \\
\hline \multirow[t]{2}{*}{ Search agents' objective } & Parochial (partial) performance & $\mathrm{x}$ & $\mathrm{x}$ & - \\
\hline & Overall performance & - & - & $\mathrm{x}$ \\
\hline Coordination mechanism & Sequential; horizontal veto; proposal & $\mathrm{x}$ & $\mathrm{x}$ & $\mathrm{x}$ \\
\hline Level of communication & $E^{C} \in\{0,1\}$ & $\mathrm{X}$ & - & - \\
\hline Error & $E^{C} \in\{0,1,2,3,4\}$ & - & $\mathrm{X}$ & $\mathrm{X}$ \\
\hline
\end{tabular}

$M$ of subproblems and agents, respectively, additional simulations for $M \in\{2,3,6\}$ were run. $^{2}$

The complexity of the search problem is captured by parameter $K$ (see Sect. 3.1). In order to describe the complexity of the task to be accomplished by the DPSS, we employ a further parameter $K^{*}$ : assume that the nature of the $N$-dimensional decision problem allows to identify $G$ subgroups of decisions $d_{i}$ with intense intra-group interactions and a level $K^{*}$ of cross-group interactions.

For example, in the perfectly decomposable structure (Fig. 2a), four subgroups can be identified with no interactions among them (i.e., $K^{*}=0$ ) which could mutually affect the partial performance achieved for other subgroups [43]. Then, in case that the assignment of subproblems to the agents mirrors the structure of the overall problem, no coordination among agents is required [4,57]. On the contrary, in the full interdependent case ( $K=11$ ) all single options $d_{i}$ affect the performance contributions of all other choices and the coordination need is maximal (Fig. 2d); should the DPSS have $M=4$ agents-as exemplary shown in Fig. $2 d$ by the shaded "areas" $-K^{*}$ equals 9. Moreover, two intermediate levels of complexity are simulated and with, for four agents $(M=4)$, one with low $\left(K^{*}=1\right)$ and one with medium level $\left(K^{*}=5\right)$ of cross-agent interactions (Fig. 2b, c).

The simulation study comprises three types of simulation experiments (for details see Table 1) with the results being introduced in Sects. 5.1 to 5.3:

Type I: First, some baseline scenarios are simulated in order to provide a basic understanding of the effects of erroneous communication. In the baseline scenarios, search problems of

2 The decompositions into $M \in\{2,3,4,6\}$ were chosen since, with $N=12$, they allow an equal range of agents' competencies; otherwise, effects of different scopes of agents' competencies could additionally effect results. 
(a) block diagonal structure $\left(K=2, K^{*}=0\right)$

Performance Contribution $C_{j}$

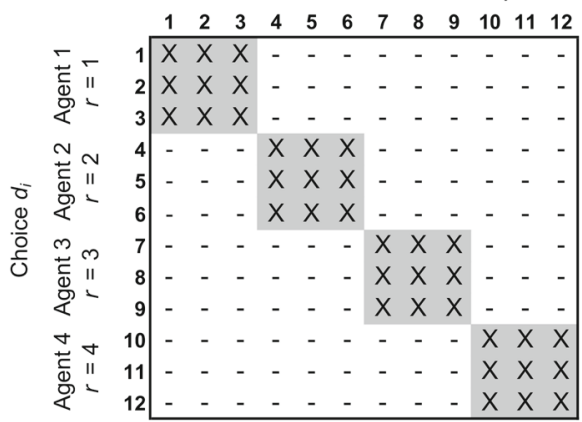

(c) intermediate structure medium $\left(K=7, K^{*}=5\right)$

Performance Contribution $C_{j}$

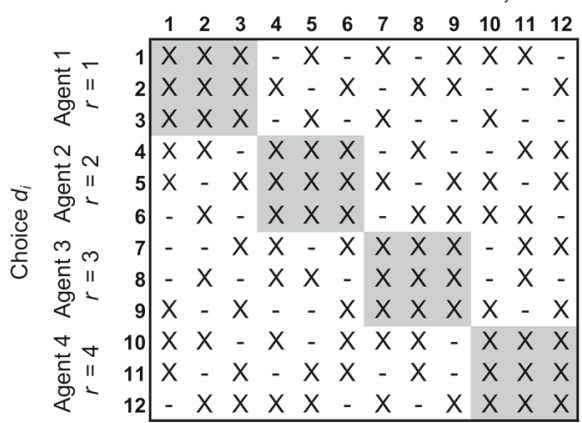

$$
\begin{array}{cl}
\text { Notes: } \\
& \text { Scope of primary control of agent } r \\
\times & \text { Choice } i \text { affects performance contribution } j \\
- & \text { Choice } i \text { does not affect performance contribution } j
\end{array}
$$

(b) intermediate structure low $\left(K=3, K^{*}=1\right)$

Performance Contribution $C_{j}$

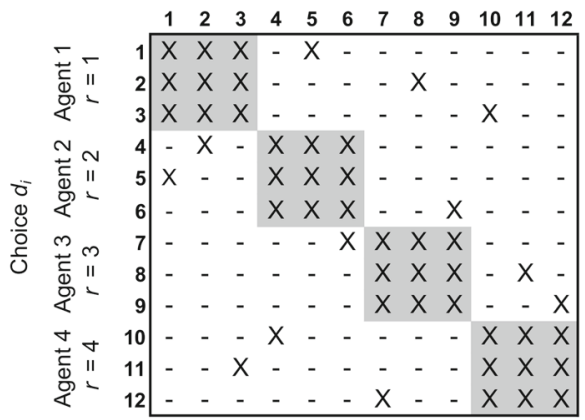

(d) full interdependent structure $\left(K=11, K^{*}=9\right)$

Performance Contribution $C_{j}$

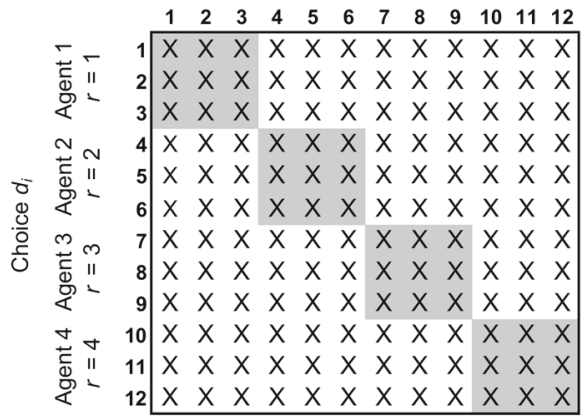

Fig. 2 Types of interaction structures in the simulation experiments. The plots show a task assignment to four agents

a nearly decomposable nature assigned to $M=4$ agents $\left(K^{*}=1\right.$, see Fig. $\left.2 b\right)$ are studied where, thus, the coordination need is at a rather moderate level. The search agents pursue a parochial objective, i.e., they seek to increase the performance obtained for their partial search problems and, hence, no coordination by objective is employed. However, the simulations are run for the three different coordination mechanisms introduced in Sect. 3.4. Results obtained with perfect communication are compared to those obtained with slightly imperfect communication of $E^{C}=1$.

Type II: The second type of simulation experiments is intended to study the sensitivity of results against the level of communication errors as well as the complexity of the search problems. Hence, for each of the three coordination mechanisms under investigation, the level of communication errors is stepwise increased up to $E^{C}=4$. In this course, also an analysis of the effects of communication errors with respect to the agents' parochial performance is provided. Moreover, in addition to the "intermediate low" structure, the other interaction structures depicted in Fig. 2 are investigated. Since also the number $M$ of agents employed shapes coordination need and communication flows, results obtained for different numbers of agents are introduced. 
Type III: The third type of experiments investigates whether and, if so, how the effects of erroneous communication in the three coordination mechanisms are affected, when additionally some coordination by objective is provided. In particular, while in the experiments of types I and II the search agents seek to increase the performance obtained for their respective partial search problem, now the search agents pursue the overall performance as an objective. Hence, an interesting question is whether with this additional kind of coordination the effects of erroneous communication are mitigated or aggravated.

\section{Results and discussion}

This section intends to introduce the results obtained from the simulation experiments. The key results are also summarized in Table 6 (Appendix) with respect to prior related work.

\subsection{Communication errors in situations with moderate coordination need (type I experiments)}

Table 2 displays condensed results obtained from the simulation experiments Type I, i.e., for the baseline scenarios. In particular, the final performance $\left(V_{t=250}\right.$, column 2$)$ achieved in the end of the observation period and the performance yielded on average in each of the 250 periods $\left(\bar{V}_{\{0 ; 250\}}\right.$, column 4$)$ as well as the frequency of how often the global maximum has been found in the final period (column 6) may serve as metrics for the search systems' coherence in terms of the solution quality achieved. ${ }^{3}$ The numbers displayed for $V_{t=250}$ and $\bar{V}_{\{0 ; 250\}}$ are the average of 2500 simulation runs. The ratio of periods in which the configuration $\mathbf{d}$ is altered compared to the previous period (column 7) may be regarded as an indicator for the diversity of search, while the frequency of periods with false-positive movements (rightmost column) gives some idea of the quality of alterations. Figure $3 a-c$ illustrates the adaptive walks in the baseline scenarios where each line represents the average of 2500 simulations.

An intuitive first conjecture might be that erroneous communication in the course of coordination is detrimental to the level of performance obtained by the DPSS. This conjecture is confirmed for the sequential mode: an error of $E^{C}=1$ leads to a loss in final performance $V_{t=250}$ of about 3.5 points of percentage compared to perfect communication.

However, the horizontal veto mode remarkably benefits from a low communication error since the final performance exceeds that obtained for perfect communication by more than 5 points of percentage; the ratio of adaptive walks which end in the global maximum of the respective performance landscapes increases from 7.64 to $20 \%$. Compared to that, the proposal mode seems to be rather robust against a slight distortion of communication.

Subsequently, in particular, the (somehow counter-intuitive) results for the horizontal veto and the proposal mode are discussed more into detail, while a discussion of the sequential mode's results is postponed to Sect. 5.2.

With respect to the horizontal veto mode, an analysis more into detail reveals that the beneficial effects of erroneous communication reasonably are caused by the effects of errors on the diversity of search: the horizontal veto mode is particularly prone to inertia, i.e., mutual blockading, since this coordination mode effectively prevents false-positive decisions from the distributed problem solvers' perspectives (see also $[17,46])$ : with each single agent

3 The final performance $V_{t=250}$ and average performance $\bar{V}_{\{0 ; 250\}}$ is given in relation to the global maximum of the respective fitness landscape: otherwise, the results could not be compared across different landscapes. 


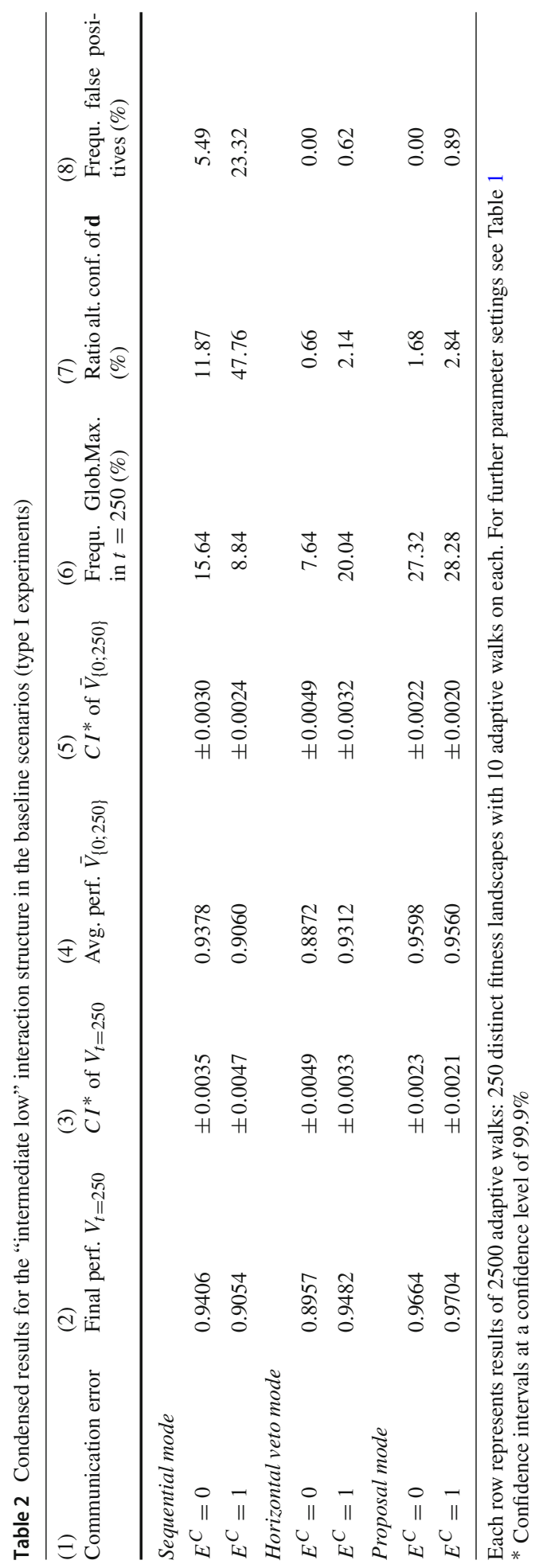


(a) sequential mode

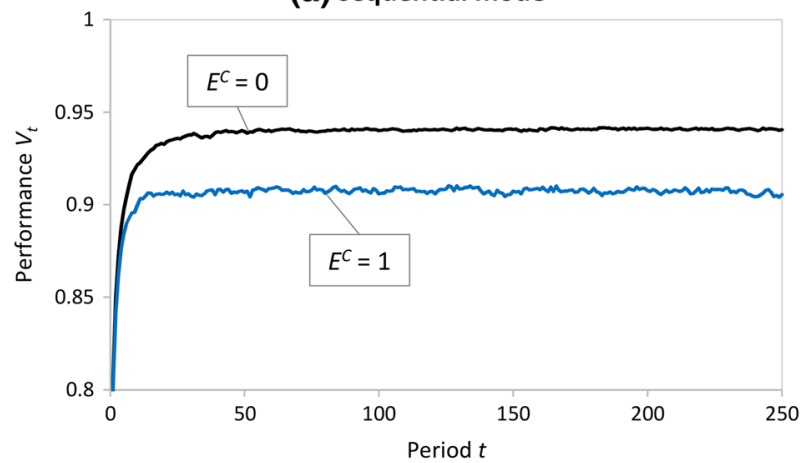

(b) horizontal veto mode

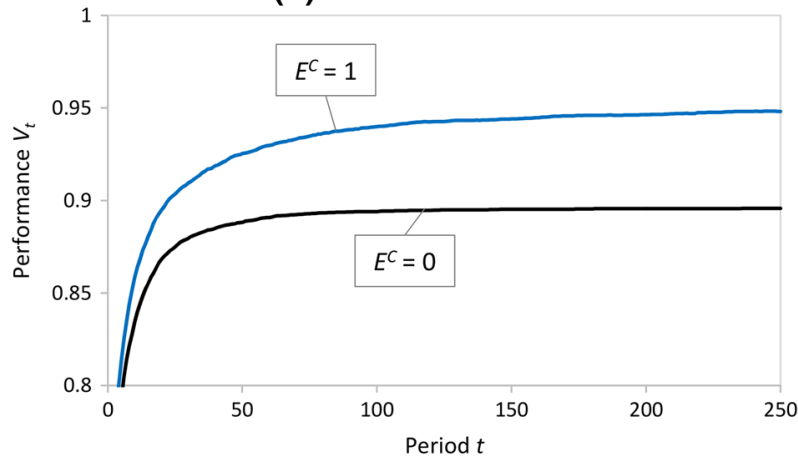

(c) proposal mode

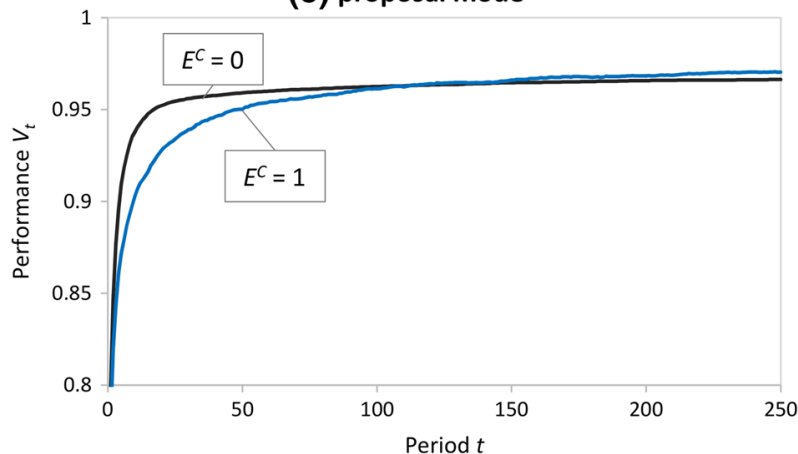

Fig. 3 Adaptive walks for the "intermediate low" interaction structure in the baseline scenarios (type I experiments). Each line represents the average of 2500 adaptive walks, i.e., 10 walks on each of 250 landscapes. For further parameter settings see Table 1

pursuing its parochial performance, no agent accepts a decrease in the "own" performance $P_{t}^{r}$ and, thus, vetoes against other agents' choices which would—due to interactions — negatively affect the "own" performance. This becomes obvious for $E^{C}=0$ where in only $0.66 \%$ of periods alterations take place (Table 2). The downside is that this precludes options to be chosen which would-at the cost of some partial performance-increase the overall performance $V_{t}$. 
With communication errors in the horizontal veto mode, there is the chance that the "parochial" rejection of overall beneficial options is reduced: when a search agent's preferred option is communicated erroneously to the fellow agents, these may come to a falsely positive or falsely negative evaluation of the effects on the own partial performance. Apparently, slightly distorted communication leads to a broader exploration of the solution space. In particular, with $E^{C}=1$ the ratio of periods in which a new configuration $\mathbf{d}$ is implemented raises to $2.1 \%$ of periods, though about one third of these alterations (i.e., in $0.66 \%$ of the 250 periods) this is in favor of false-positive movements. ${ }^{4}$ However, these findings correspond to results of prior research indicating that errors (in the assessment of options) could be beneficial [54].

The results obtained for the proposal mode, so far, show some similarities to those of the horizontal veto mode: with slightly distorted communication the diversity of search increases, though at a rather low level and at the cost of a third of the alterations being in favor of false-positive configurations. However, in sum, the effect of the low communication error on final and average performance appears negligible in the proposal mode. An interesting question is whether the apparent trade-off between increased diversity and reduced quality of search is shifted when the level of communication errors increases or the complexity of the $N$-dimensional problem is varied. This is studied in the subsequent section.

\subsection{Sensitivity to communication errors and problem complexity (type II experiments)}

In order to study, the sensitivity of coordination mechanisms to erroneous communication in differently complex environments, two types of comparisons are introduced: (1) the final performance $V_{t=250}$ achieved with imperfect communication is compared to that obtained with perfect communication for each coordination mechanism and each level of complexity under investigation; (2) for given levels of communication error and complexity, the final performances $V_{t=250}$ achieved with different coordination mechanisms are compared pairwise against each other. The former comparison directly indicates on the sensitivity on communication errors and the latter reports on the relative advantages of the three coordination mechanisms and allows to identify the contextually best performing coordination mechanism.

In particular, with respect to the first comparison, i.e., coordination mechanisms' sensitivity to erroneous communication, the mean differences of the final performance $V_{t=250}$ obtained for an error level $E^{C}>0$ against that for $E^{C}=0$ are of particular interest. Figure 4 plots the mean differences of final performances for each interaction structure and for each level of communication error under investigation; Table 3 in the Appendix reports on the mean differences in final performances and significances more into detail.

Regarding the coordination mechanisms' sensitivity to erroneous communication, the results displayed in Fig. 4 broadly, though not universally, support intuition that communication errors occurring in the course of coordination reduce the overall systems' performance. Moreover, the results suggest that the performance losses compared to perfect communication remarkably depend on the coordination mode employed and on the complexity of the search problem. In particular, the results suggest that with increasing coordination need among

\footnotetext{
4 Simulation experiments with an observation period of $T=500$ yield similar results: the final performance achieved with $E^{C}>0$, in average, goes about 5 points of percentage beyond that level obtained with perfect communication; in $0.33 \%$ of the 500 periods with $E^{C}=0$ new solutions are implemented; for $E^{C}>0$ this ratio goes up to $1.6 \%$.
} 
(a) block-diagonal structure $\left(K=2, K^{*}=0\right)$

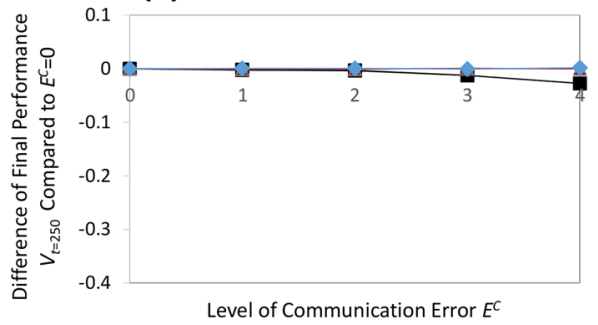

(c) intermediate structure medium $\left(K=7, K^{*}=5\right)$

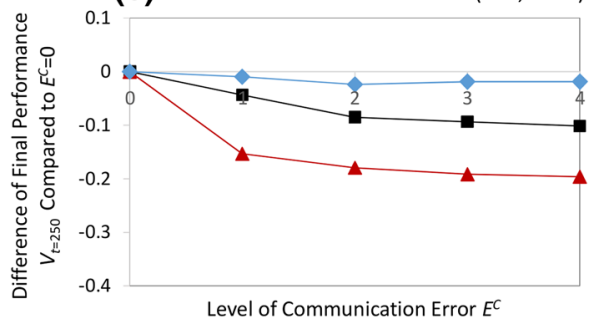

$\longrightarrow$ sequential (b) intermediate structure low $\left(K=3, K^{*}=1\right)$

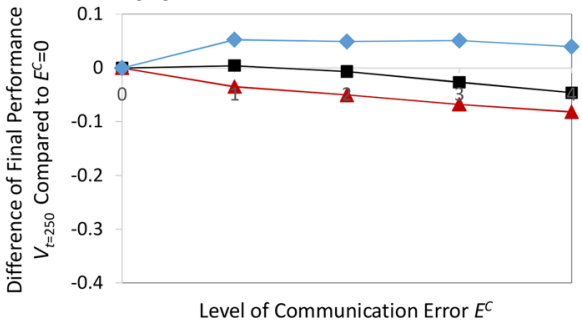

(d) full interdependent structure $\left(K=11, K^{*}=9\right)$

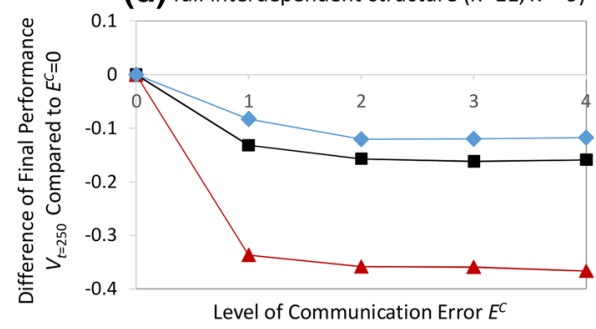

horizontal veto $\longrightarrow$ proposal

Fig. 4 Differences of means of final performance achieved with communication error of level $E^{C}$ compared to perfect communication $\left(E^{C}=0\right)$ with search agents pursuing parochial performance. Each mark represents the average of 2500 adaptive walks, i.e., 250 distinct fitness landscapes with 10 adaptive walks on each. For parameter settings see Table 1

agents as captured by $K^{*}$, the performance losses obtained with erroneous communication increase too. However, some aspects deserve to be analyzed more into detail.

First, in case that there is no need for coordination among agents (i.e., in the block-diagonal structure, where $K^{*}=0$ for $M=4$ ), communication errors reduce only in the proposal mode slightly the final performance achieved, whereas with the other coordination modes no negative performance effects occur. The (rather low) performance losses in the proposal mode result from erroneous rejections of the agents' most preferred options-based on a falsely communicated preferences-by the central agent: without cross-agent interactions (i.e., $K^{*}=0$ ) the agents' preferences also reflect the best possible options with respect to the overall performance; however, due to distorted communication it might be that the central agent rejects the solution composed of search agents' first preferences.

Second, the horizontal veto mode does not only benefit from even high error levels in case of low coordination need (i.e., for $K^{*}=1$ for $M=4$ ); moreover, for higher coordination needs the horizontal veto mode is the least sensitive to communication errors. The mutual veto power, prevents that falsely communicated options are accepted which, for higher coordination prevents performance losses. A closer analysis reveals that, for example, in the full interdependent structure (i.e., $K^{*}=9$ for $M=4$ ), the diversity of search, as captured by the ratio of new configurations $\mathbf{d}$ implemented, ranges around $2.5-3 \%$, and only $1-1.5 \%$ of the periods show false-positive movements (in the proposal mode these indicators are around 21 and $10 \%$, respectively, and for the sequential mode they range around 97 and $48 \%$, respectively).

Third, while for problem structures implying a coordination need (i.e., when $K^{*}>0$ ) performance losses in the proposal mode are at a medium level, the sequential mode is the 
(a) sequential mode

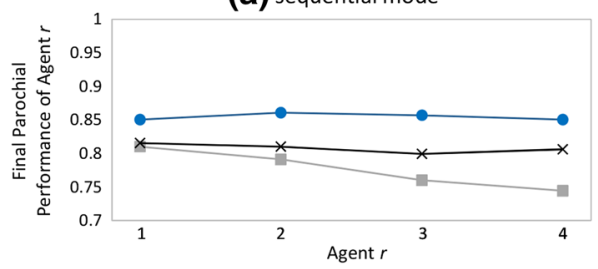

(c) proposal mode

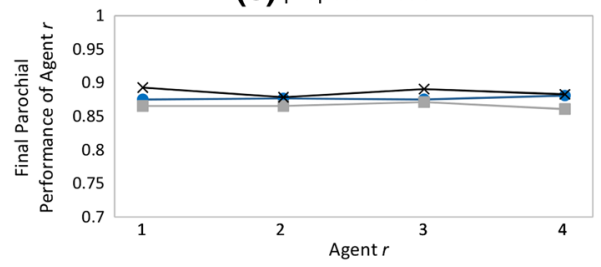

(b) horizontal veto mode

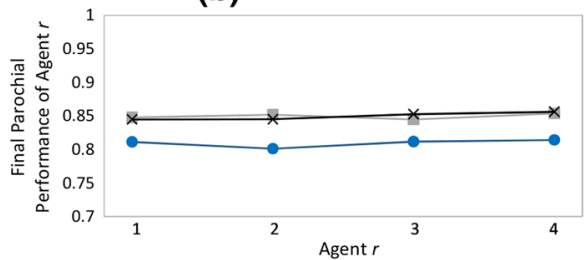

with

Level $E^{C}$ of Communication Error

$\longrightarrow 0$

$\longrightarrow 2$

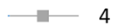

Fig. 5 Parochial performance achieved in respect to the agents' subproblems for an interaction structure with moderate coordination need. Parochial performances are given normalized to the local optima of the respective subproblems. Each mark represents the average of 2500 adaptive walks, i.e., 250 distinct fitness landscapes with 10 adaptive walks on each. For further parameter settings see Table 1

most sensitive to communication errors: the maximal loss is 37 points of percentage which occurs in case of full interdependence.

Hence, an interesting question is what might cause the sequential mode's low robustness against imperfect communication. I argue that the propagation of errors incorporated in this mode may provide the explanation. For example, an error occurring in the communication of agent 1's plan is propagated to all subsequent agents; not only does agent 2 adjust its partial plan to the distorted plan of agent 1; next, agent 3 takes the imperfectly communicated plan of agent 1 and the erroneously adjusted and, perhaps additionally, imperfectly communicated plan of agent 2 into consideration and so on. This propagation of errors and the mutual adjustment of subsequent plans to erroneously communicated previous choices are irrelevant if no interdependencies among agents exist (i.e., for $K^{*}=0$ ) and become rather detrimental for maximal complexity (i.e., $K^{*}=9$ ) and with increasing levels of communication errors.

This explanation is supported by an analysis of the agents' parochial performances $P_{t}^{r}\left(\mathbf{d}_{t}^{r}\right)$ (Eq. 3) as exemplarily displayed in Fig. 5 for the "intermediate low" interaction structure (Fig. 2b) for different levels of communication error. ${ }^{5}$ The horizontal veto mode and the proposal mode do not show any notable performance differences among the agents' performances for a given error level. In contrast, with a highly erroneous communication, in the sequential mode the performance declines with the sequence in the mutual adjustment, i.e., the last agent achieves the lowest parochial performance. ${ }^{6}$

The second type of comparisons presented in this section is intended to give some evidence on the contextually best performing coordination mechanism where the context is characterized by the level of communication errors and the complexity of the search prob-

\footnotetext{
5 The parochial performances achieved are given in relation to the local optima of the respective subproblems: otherwise, the results could not be compared across different performance landscapes.

6 Normalized parochial performances partially are remarkably below the level obtained for the respective overall performances (see Table 2). This is a result of coordination among agents' actions meaning that-due to the interactions among the agents' partial problems-superior solutions to the overall problem $d$ do not necessarily go along with superior solutions for the partial problems $d^{r}$.
} 
lem. For this, the differences in means of final performance $V_{t=250}$ obtained with different coordination mechanisms and differentiated for the context are computed and depicted in Fig. 6. The pairwise comparisons provide some interesting findings:

First, the results confirm intuition that for perfectly decomposable structures neither the particular coordination mode nor the level of communication errors is of major relevance: apart from the slight sensitivity of the proposal mode to distorted communication (see above), as expected, no remarkable performance differences are apparent. Moreover, results suggest that, in tendency, with higher levels of complexity the mutual relative (dis-)advantages of coordination mechanisms increase.

Second, with the transition from perfect to slightly distorted communication (i.e., transition from $E^{C}=0$ to $E^{C}=1$ ) the relative (dis-)advantages among coordination mechanisms show the most remarkable changes. This appears most strikingly in the comparison of the horizontal veto against the sequential mode: for $E^{C}=0$, the sequential mode outperforms the horizontal veto mode by up to 15 points of percentage and, with $E^{C} \geq 1$, things change: then the horizontal veto mode outperforms sequential planning by up to 12 point of percentage. For $E^{C} \geq 1$, the performance differences between the coordination modes appear relatively stable across different levels of communication errors. This suggests that, in tendency, if communication does not work perfectly, then the level of erroneous communication in the vast majority of cases ${ }^{7}$ does not affect the relative (dis)advantages of the coordination mechanisms over each other.

Third, for nearly all situations implying some coordination need (i.e., for $K^{*} \geq 1$ ) and with erroneous communication, the proposal mode yields the highest final performance. This provides some evidence for employing central decision-making authority - in particular, when communication may not work perfectly, when complexity of the overall problem to be solved is high or when even the task assignment does not mirror the interaction structure, for example, since it is not known in advance.

With respect to the latter aspect, simulations conducted for DPSS with different numbers of agents show that the proposal mode is particularly robust against decompositions into subproblems and respective task assignments which do not precisely mirror the nature of the problem: Fig. 7 reports the final performances $V_{t=250}$ obtained for different numbers of agents ${ }^{8}$ in the "intermediate low" interaction structure as indicated in Fig. $2 b$ with $K=3$. It should be stressed that with an $M \neq 4$ the decomposition does not exactly mirror the "nature" of that decision problem [4].

Moreover, the number of agents affects the ratio of the $N$ dimensions of the decision problem that could be altered in one time step: with respect to the assumptions on limited information-processing capabilities of agents (see Sect. 2), in the model the number of newly discovered options per agent and period is restricted (Sect. 3.2). However, with an increasing (decreasing) number of agents the ratio of the $N$ dimensions of the decision problem that could be altered in one time step increases (decreases) too. Hence, with an increasing (decreasing) number of agents, performance enhancements may be more (less) feasible [57]. On the other hand, with increasing (decreasing) number of agents, the peril of miscommunication may increase (decrease) with its effects on decision-making. Hence, an interesting trade-off between effects potentially enhancing and reducing performance appears.

\footnotetext{
${ }^{7}$ Only for $K^{*}=1$ the order between the horizontal veto and the proposal mode changes with increasing communication error.

${ }^{8}$ Apart from the decomposition into $M=4$ subproblems (see Table 1), the following decompositions leading to equal-sized subproblems are simulated: $M=2$ with agent $1: \mathbf{d}^{\mathbf{1}}=\left(d_{1}, \ldots, d_{6}\right)$ and agent 2: $\mathbf{d}^{\mathbf{1}}=\left(d_{7}, \ldots, d_{12}\right) ; M=3$ with agent $1: \mathbf{d}^{\mathbf{1}}=\left(d_{1}, \ldots, d_{4}\right)$, agent $2: \mathbf{d}^{\mathbf{2}}=\left(d_{5}, \ldots, d_{8}\right)$, agent 3 : $\mathbf{d}^{\mathbf{3}}=\left(d_{9}, \ldots, d_{12}\right)$ and $M=6$ with agent $1: \mathbf{d}^{\mathbf{1}}=\left(d_{1}, d_{2}\right)$ to agent $6: \mathbf{d}^{\mathbf{6}}=\left(d_{11}, d_{12}\right)$
} 
(a) horizontal veto vs. sequential mode

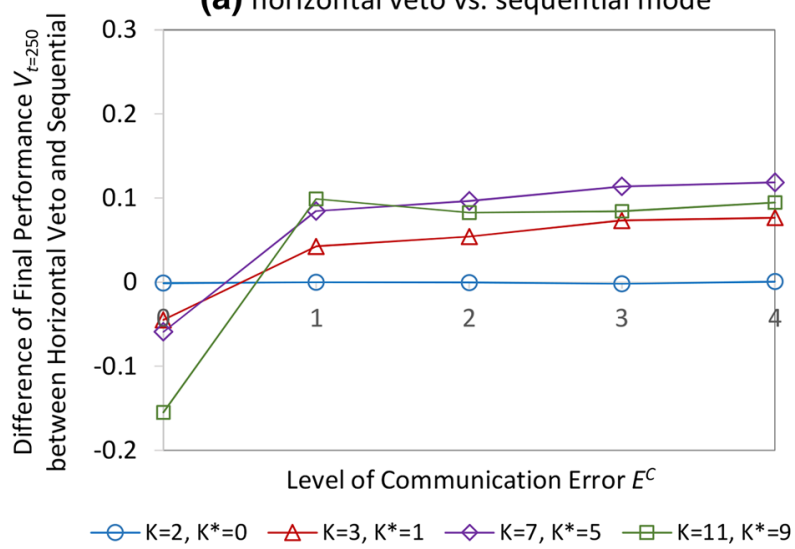

(b) proposal vs. sequential mode
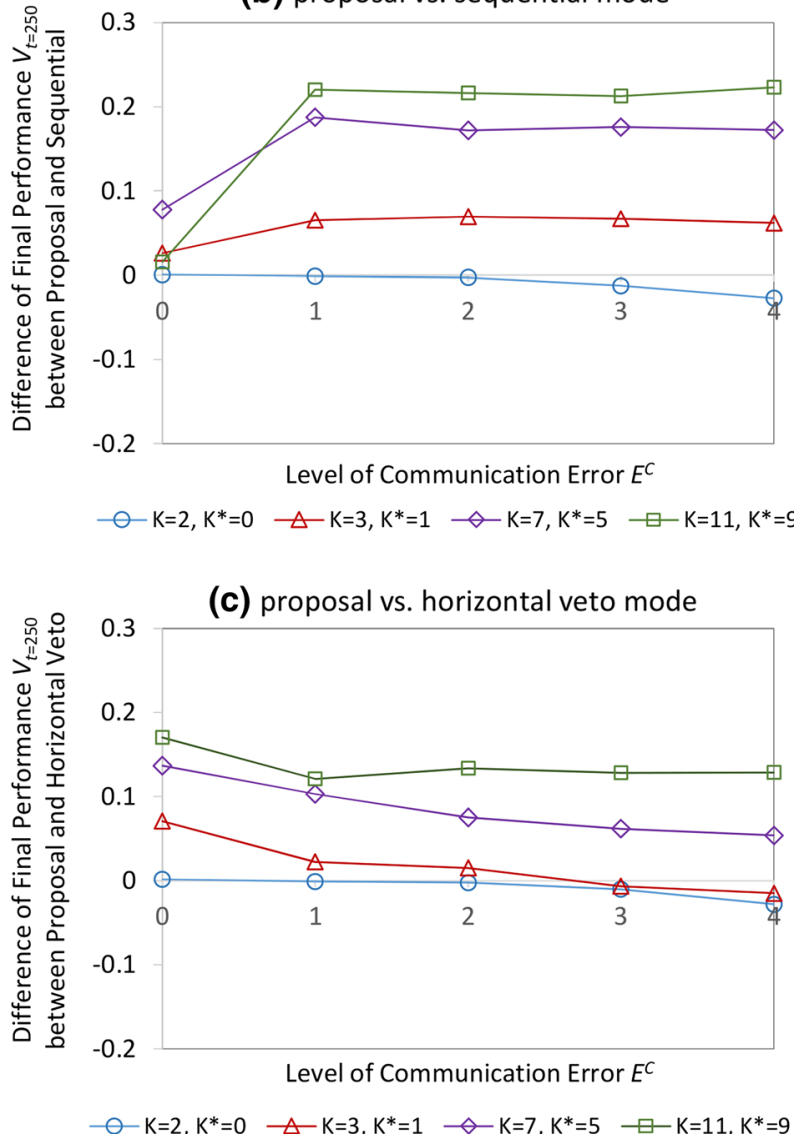

Fig. 6 Pairwise comparisons of final performance achieved with sequential, horizontal veto and proposal mode for different levels of erroneous communication and problem complexity with search agents pursuing parochial performance. Each mark represents the difference between the averages of 2500 adaptive walks, i.e., 250 distinct fitness landscapes with 10 adaptive walks on each. For further parameter settings see Table 1 
(a) sequential mode

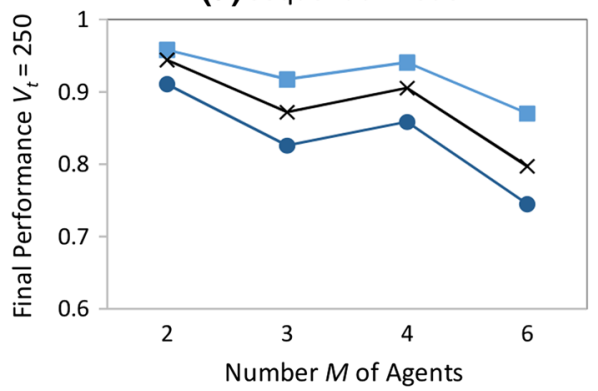

(c) proposal mode

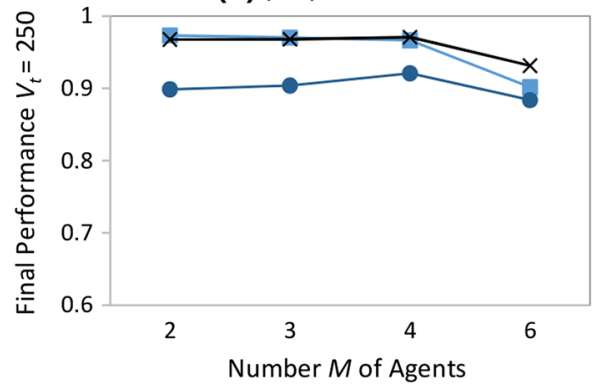

(b) horizontal veto mode

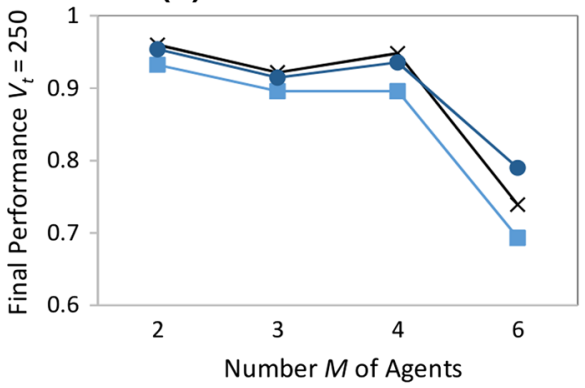

with Level $E^{C}$ of Communication Error

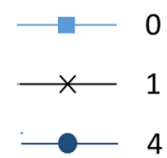

Fig. 7 Final performance achieved with DPSS comprising different numbers $M$ of agents for an interaction structure with moderate coordination need. Each mark represents the average of 2500 adaptive walks, i.e., 250 distinct fitness landscapes with 10 adaptive walks on each. For further parameter settings see Table 1

The results suggest that "overdecomposition" (i.e., $M=6$ ) is particularly detrimental which is in line with prior research [4,20,34,41,57]. However, apart from the case of $M=6$, for a given level of communication error, performances obtained appear rather robust against the number of agents employed-with the proposal mode being particularly insensitive even in case of highly erroneous communication.

These findings in favor of central decision-making authority correspond to the seminal paper of Sah and Stiglitz [44]: Since in a hierarchy an option has to be positively evaluated twice to be accepted, it is less likely that options are accepted which better should have been rejected, i.e., hierarchies reduce false-positive judgments ${ }^{9}$ compared to decentralized decision-making.

\subsection{Sensitivity in case of overall performance as agents' objective (type III experiments)}

The third part of this study intends to explore the effects of erroneous communication on different coordination mechanisms in combination with coordination by objective. In particular, the results subsequently introduced relate to search agents seeking to maximize the overall performance achieved by the DPSS rather than the performance of their partial subproblem

\footnotetext{
9 The results obtained in this simulation study are in line with the findings of Sah and Stiglitz [44]: when communication is imperfect, the ratio of false-positive alterations of configurations $\mathbf{d}$ with sequential planning is about five to twelve times as high as in the proposal mode
} 
(a) block-diagonal structure $\left(K=2, K^{*}=0\right)$

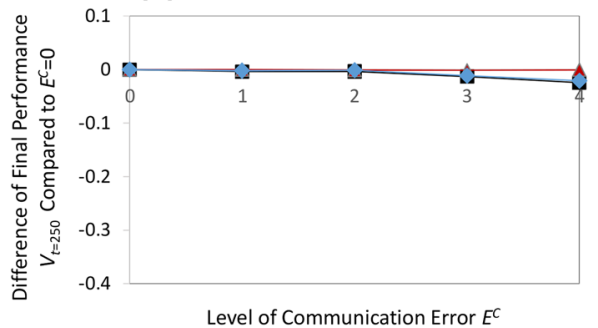

(c) intermediate structure medium $\left(K=7, K^{*}=5\right)$

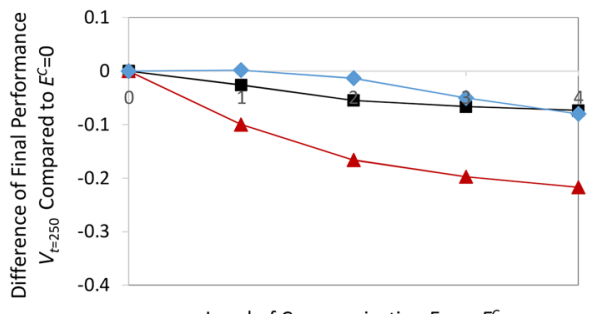

Level of Communication Error $E^{C}$ (b) intermediate structure low $\left(K=3, K^{*}=1\right)$

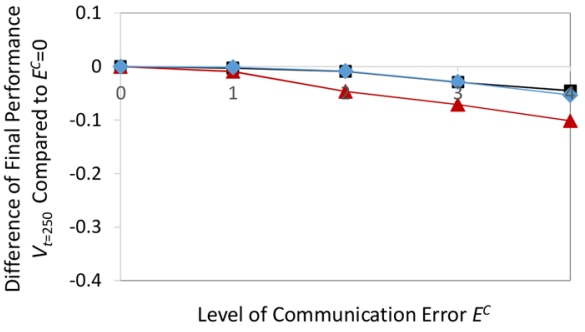

(d) full interdependent structure $\left(K=11, K^{*}=9\right)$

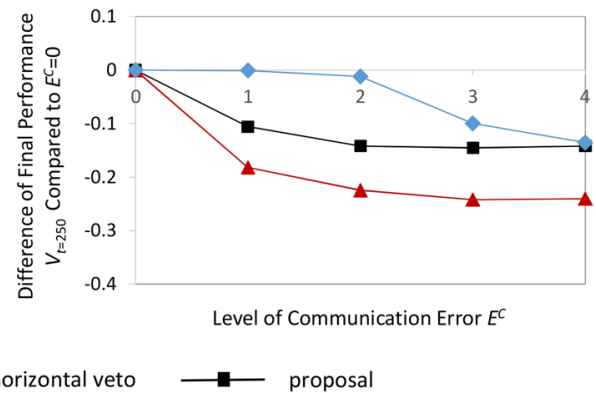

Fig. 8 Differences of means of final performance achieved with communication error of level $E^{C}$ compared to perfect communication $\left(E^{C}=0\right)$ with search agents pursuing overall performance. Each mark represents the average of 2500 adaptive walks, i.e., 250 distinct fitness landscapes with 10 adaptive walks on each. For parameter settings see Table 1

like in the previous sections. For this, analyses corresponding to the previous section are run: Fig. 8 plots the mean differences in final performance obtained with perfect compared to imperfect communication and Table 4 (Appendix) reports on the mean differences and their significance into detail; Figure 9 displays the results of pairwise comparisons among the coordination mechanisms for different levels of communication errors and complexity.

An interesting question is whether the additional component of coordination affects the sensitivity of the coordination mechanisms under investigation to communication errors: the intuition behind is that, if search agents consider the overall performance in their formation of preferences, this could decrease detrimental effects of the partial choices on the overall objective [45] and, thus, also may make coordination among agents less prone to communication errors. Hence, this analysis also contributes to the discussion on the combination of different types of coordination ("multiple controls") [5,26].

The results provide broad though not universal support for these conjectures: as is displayed in Table 5 (Appendix), in the vast majority of cases with coordination need (i.e., when $K^{*}>0$ ) the mean of final performance obtained with additional coordination by objective significantly goes beyond the level achieved with search agents pursuing merely parochial performance. These results broadly correspond to prior research-obtained with different methods such as closed-form analysis, simulation as well as empirical studies (e.g., $[7,46,56])$.

Hence, this suggests that combining different types of coordination may be an appropriate way to increase performance-even in case of erroneous communication. However, with respect to the sensitivity to erroneous communication results provide no unequivocal direction 
(a) horizontal veto vs. sequential mode

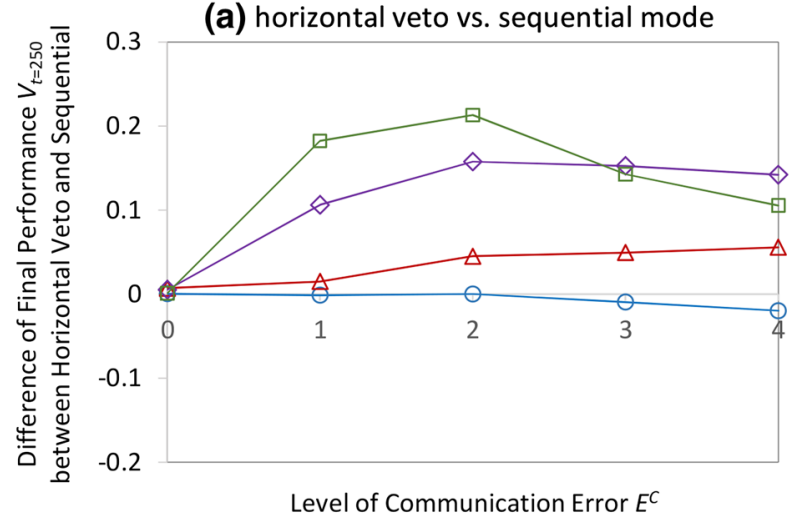

$\ominus-K=2, K^{*}=0 \triangle K=3, K^{*}=1 \leadsto-K=7, K^{*}=5 \quad \square-K=11, K^{*}=9$

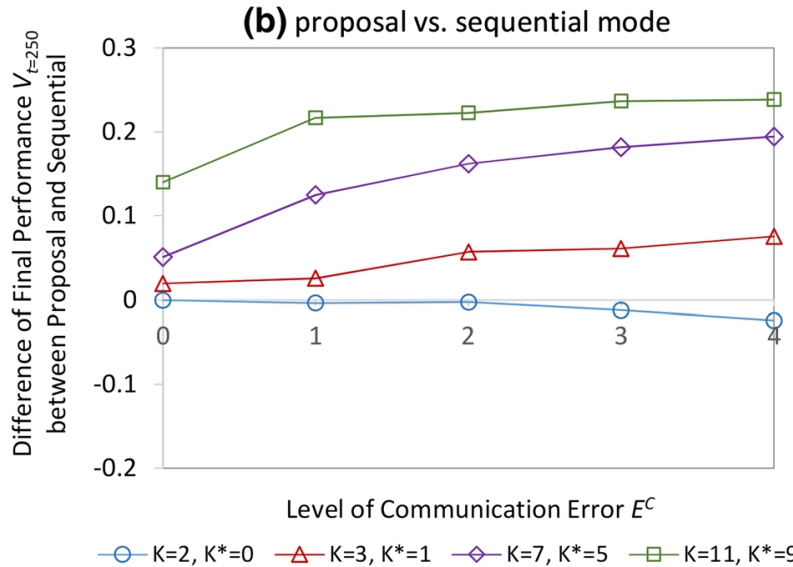

(c) proposal vs. horizontal veto mode

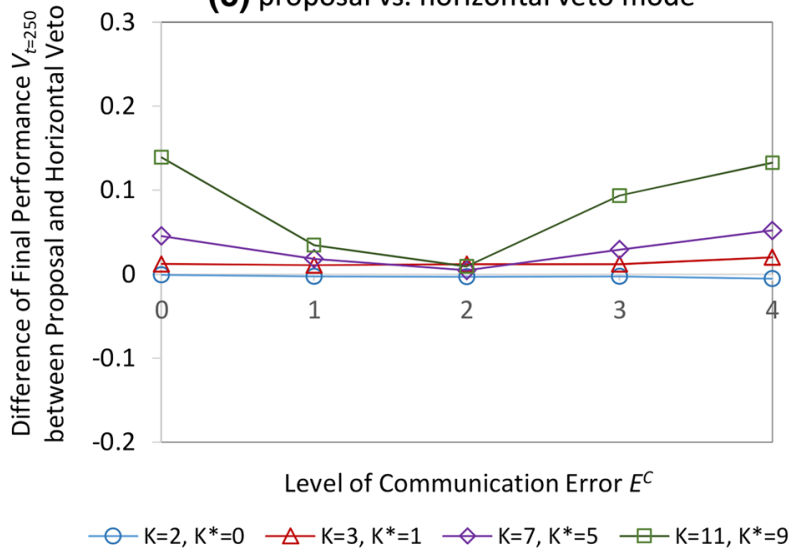

Fig. 9 Pairwise comparisons of final performance achieved with sequential, horizontal veto and proposal mode for different levels of erroneous communication and problem complexity with search agents pursuing overall performance. Each mark represents the difference between the averages of 2500 adaptive walks, i.e., 250 distinct fitness landscapes with 10 adaptive walks on each. For further parameter settings see Table 1 
of effects of the additional coordination component and deserve some closer analysis and comparison to the results of the previous experiments.

Without any need for coordination, i.e., in the block-diagonal structure, performance effects of erroneous communication in all three coordination modes are at rather low level. With increasing problem complexity, in tendency, the effects of communication errors increase. These results correspond to the results of the previous section (see Fig. 8a-d compared to the respective plots in 4).

However, the results obtained for the horizontal veto mode deserve closer attention since some differences compared to the respective experiments with parochial goals appear. The contrast is rather obvious for the "intermediate low" structure where, for parochial goals, communication errors turned out to have beneficial effects whereas, now, higher levels of communication errors are detrimental. In contrast, now, with the horizontal mode even an communication error of $E^{C}=2$ does not lead to considerable performance losses when complexity is maximal (Fig. 8d). A closer analysis reveals that this might be caused by the different effects that communication errors have, in particular, on the diversity of search when the veto mode is combined with coordination by objective. The reason for these diverging effects of erroneous communication may lie in the particular deficiencies of the veto mode which is, in general, the peril of inertia [46].

A starting point for an analysis is the situation with perfect communication: when search agents pursue parochial performance, the search processes are particularly prone to inertia since the search agents evaluate the "overall" option only with respect to their own "narrow" objective. When, in contrast, search agents pursue overall performance, for example, reductions in the "own" performance could be compensated by higher performance related to the "rest" of the search problem. Hence, with overall performance pursued, the criterion in Eq. 6 is, in fact, less rigid. This makes mutual vetoes less likely, and, thus, more alterations occur which leads to a higher final performance. As argued in the previous section, for parochial goals pursued, communication errors increase false-positive alterations-i.e., movements in favor of options which would have been vetoed with perfect communication-but, by that, reduce inertia (see Table 2). In contrast, when search agents pursue overall performance, some kind of tipping point behavior occurs at a level of $E^{C}=2$ (most obvious in Figs. $8 \mathrm{~d}$ and 9c). Apparently, when combined with coordination by objective, the horizontal veto mode can cope rather well with a moderate level of communication error (see the rather low performance losses for $E^{C}=1$ and $E^{C}=2$ ); beyond that level, performance losses increase - and that the more the higher the problem's complexity. I argue that this is since for higher levels of $E^{C}$ the peril of both false negative and false-positive evaluations increases. This is supported by a closer analysis which shows that, for example, in the full interdependent structure, for $E^{C}>2$ the frequency of alterations does not differ remarkably from lower levels of communication errors, while the ratio of false-positive evaluations doubles and the final performance drops by 10 points of percentage and more (see also Table 4).

With respect to the relative order among the three coordination mechanisms for different levels of communication errors and complexity (see Fig. 9), the simulations provide some similar results as yielded by the previous experiments: with respect to sequential planning, results displayed in Table 4 (see Appendix) suggest that for high complexity, like in the type II experiments, the level of final performance goes down to nearly 50 points of percentage which means that the search processes do not provide much more than a random selection of configurations. When search agents pursue overall performance, again, the proposal mode and the horizontal mode outperform sequential planning. Moreover, like in the type II experiments, in the majority of cases the proposal mode provides better results than the horizontal 
mode. This, again, provides some evidence in favor of central decision-making authority in case of erroneous communication (see 5.2 and [44]).

\section{Conclusion and future work}

This section intends to summarize the major results of this simulation study, including to highlight relations to prior research, and to suggest directions for future research. For a structured overview, Table 6 in the Appendix summarizes the key results obtained with references to related work as they are summarized subsequently.

In this study, different effects of erroneous communication on mechanisms employed for the coordination of plans of autonomous agents ("plan merging") are investigated. A major finding is that effects of erroneous communication subtly differ with the coordination mechanism employed and the level of complexity of the task to be solved collaboratively.

In particular, the results suggest that sequential planning, which provides high levels of performance when communication works perfectly, due to the built-in propagation of errors is particularly prone to imperfect communication. This supports doubts on the practical usability of the sequential coordination mode (e.g., $[12,18]$ ). In contrast, granting lateral veto power to autonomous agents, in general, shows the lowest sensitivity to erroneous communication; for low levels of coordination need, system's performance even may benefit from communication errors when lateral veto power is employed. In a more general sense, this relates to prior research, indicating that information errors may have performance enhancing effects since they increase the diversity of search (e.g., [54,55] with further references). The results further suggest that employing a central planning agent for coordination is beneficial, in particular, when the level of task complexity is at a higher level and imperfect communication is to be expected. While the contribution of centralized planning is well recognized when task complexity is high (e.g., [46]) and information is imperfect [44], this study puts particular focus on imperfections resulting from erroneous communication.

Moreover, the findings indicate that additionally employing some coordination by objective in the sense that search agents are motivated to pursue the overall system's performance rather than their sub-systems' performance is beneficial when communication is imperfect and coordination need is high. While it is well known that providing incentives directed towards the overall rather than the parochial objective to decentralized agents is beneficial when the complexity of the overall task is high (e.g., [7,56]), according to our results, this also holds when communication is erroneous.

The findings obtained in this study may be of particular interest for the organizational design of - error tolerant-distributed problem-solving systems, may they be business organizations or fleets of robots. In particular, for situations when notable communication errors may occur, findings suggest to also employ a central planning authority and to give incentives to decentralized agents related to the overall system's performance.

However, it is worth mentioning that both (1) establishing a central decision-making agent and (2) the additional coordination by objective reasonably do not come without costs: the former requires, depending on the context, for example, to establish a headquarter with appropriate capacities for information processing in a business organization or a central unit in a swarm of unmanned aerial vehicles. The latter requires that the decentralized agents are informed about the spillover effects of their choices, i.e., on the "rest" of the overall problem. Depending on the context, this may, for example, require to hire more experienced 
managers, invest in better information systems or enhance the knowledge base and decisionmaking algorithms provided to robots.

This leads to the future work for extending the study introduced: First of all, the study awaits its transfer to more practical applications in real-world problems. This will reasonably also include to increase the number and heterogeneity of agents, enrich the organizational arrangements the agents are acting in and incorporate the costs of coordination. In this vein, the findings of the paper could be subject of empirical and experimental investigations. For example, it appears worthwhile to study whether sequential planning — which is of particular relevance in corporate planning - is that prone to the propagation of errors as our results suggest or whether additional error-detecting and error-correcting mechanisms interfere. These could be of cognitive nature when individuals receive erroneously communicated plans or comprise certain organizational mechanisms for approving the planning steps.

Second, this study focuses on content-related, unintentional communication errors; however, further "types" of imperfections in communication could additionally affect the quality of communication: not only that communication may be delayed or intentionally distorted as mentioned in Sect. 2; it may also be directed to the wrong addressees or errors may occur at the recipients' site which would require to study heterogeneously imperfect versions of communicated information.

Moreover, it was beyond the scope of this paper to investigate the interaction of erroneous communication with further informational imperfections. In particular, in this study the agents are assumed to be capable of perfectly ex-ante assessing the performance contributions of options without any errors - apart from those resulting from imperfect information about other agents' preferences.

Hence, an interesting extension of this study could integrate further imperfections of communication and imperfect information processing. This might contribute to a more comprehensive understanding of the effects of imperfect information and communication in distributed planning.

Acknowledgements Open access funding provided by University of Klagenfurt.

Open Access This article is distributed under the terms of the Creative Commons Attribution 4.0 International License (http://creativecommons.org/licenses/by/4.0/), which permits unrestricted use, distribution, and reproduction in any medium, provided you give appropriate credit to the original author(s) and the source, provide a link to the Creative Commons license, and indicate if changes were made.

\section{Appendix}

Tables 3, 4 and 5 display results of comparisons of means in terms of differences between means and the significances of these differences. For this, the Welch's method [59] is employed as suggested for the analysis of simulation experiments (e.g., [31]). Table 6 summarizes the results of this study in relation to prior research. 


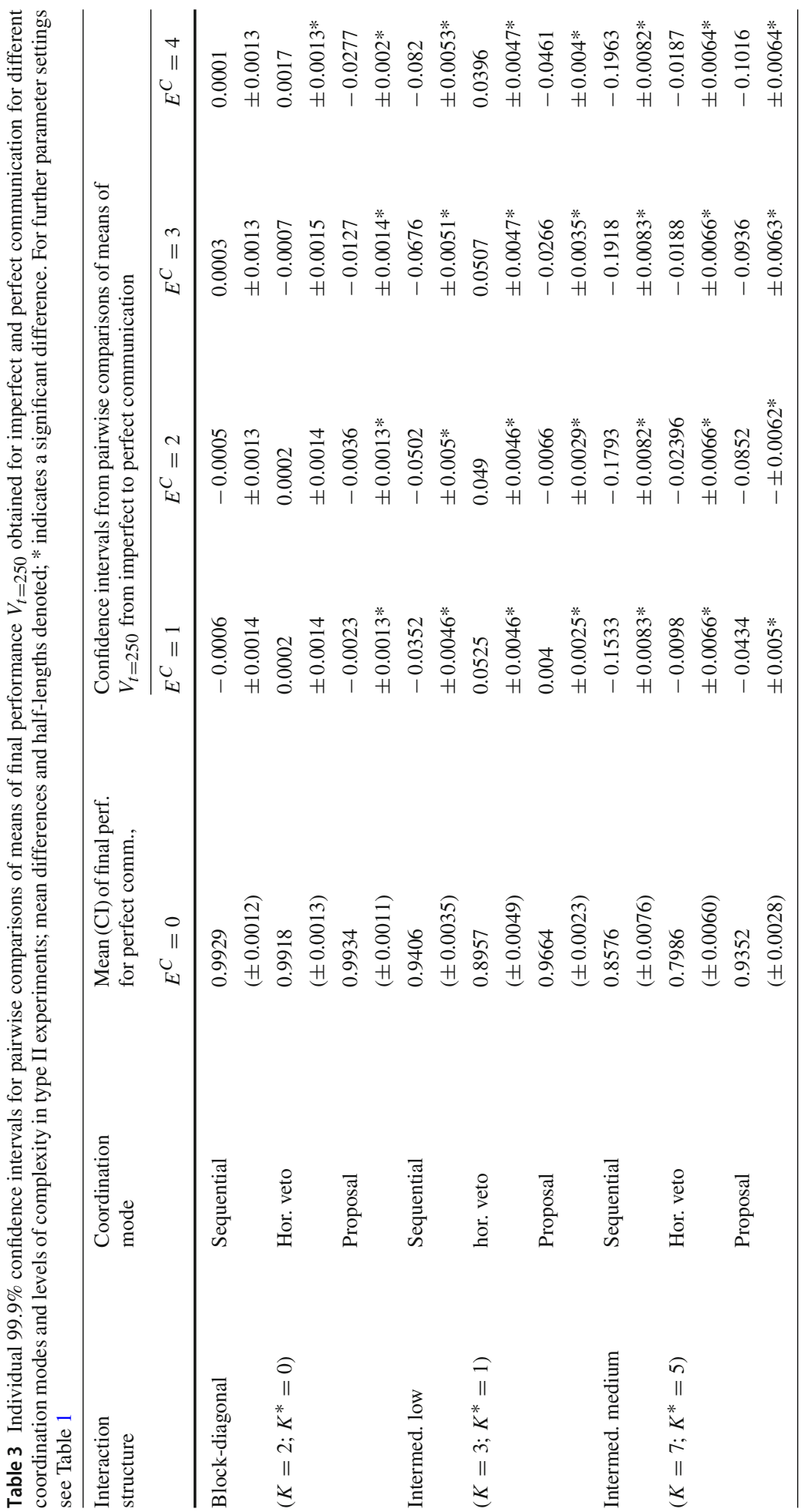




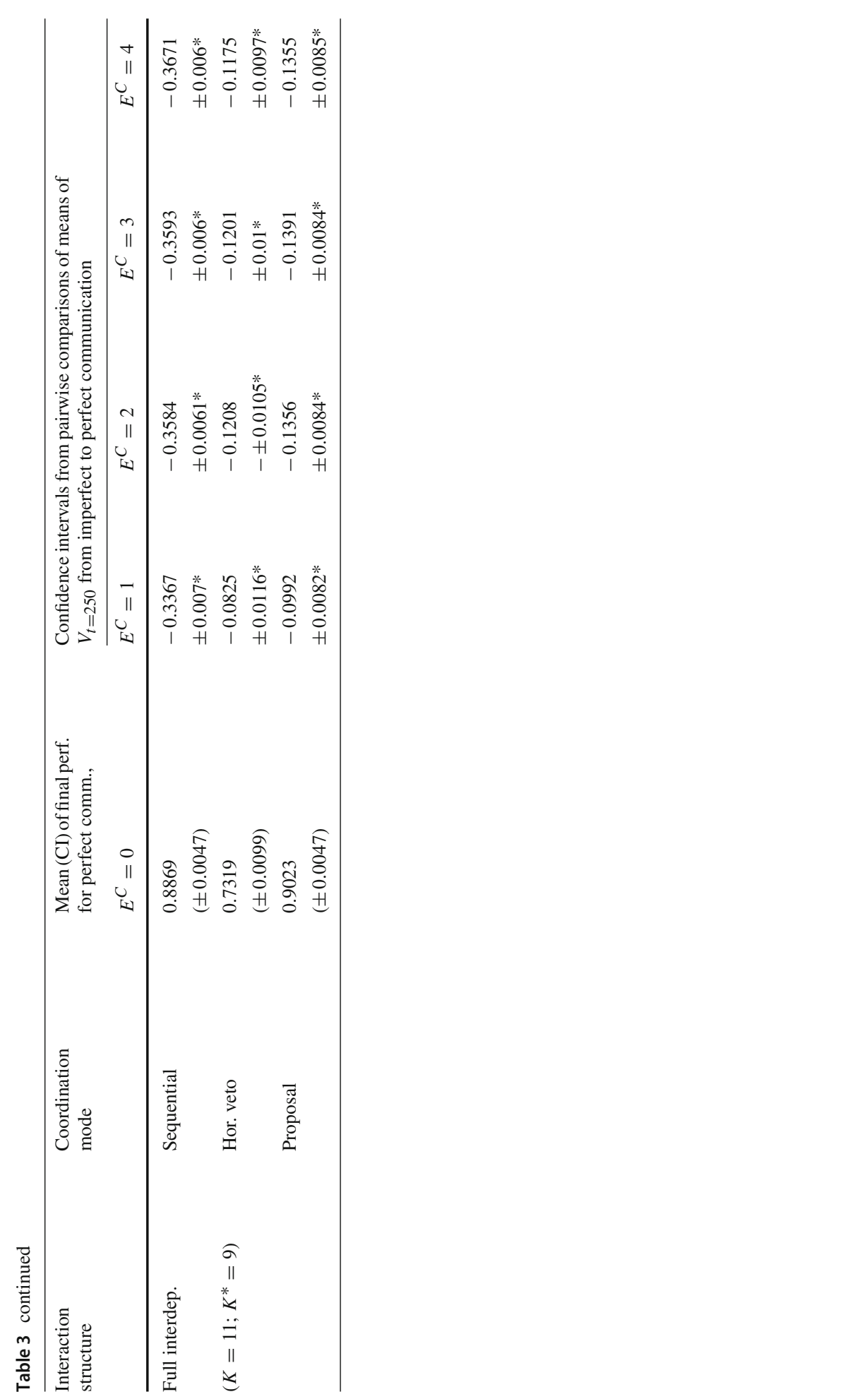




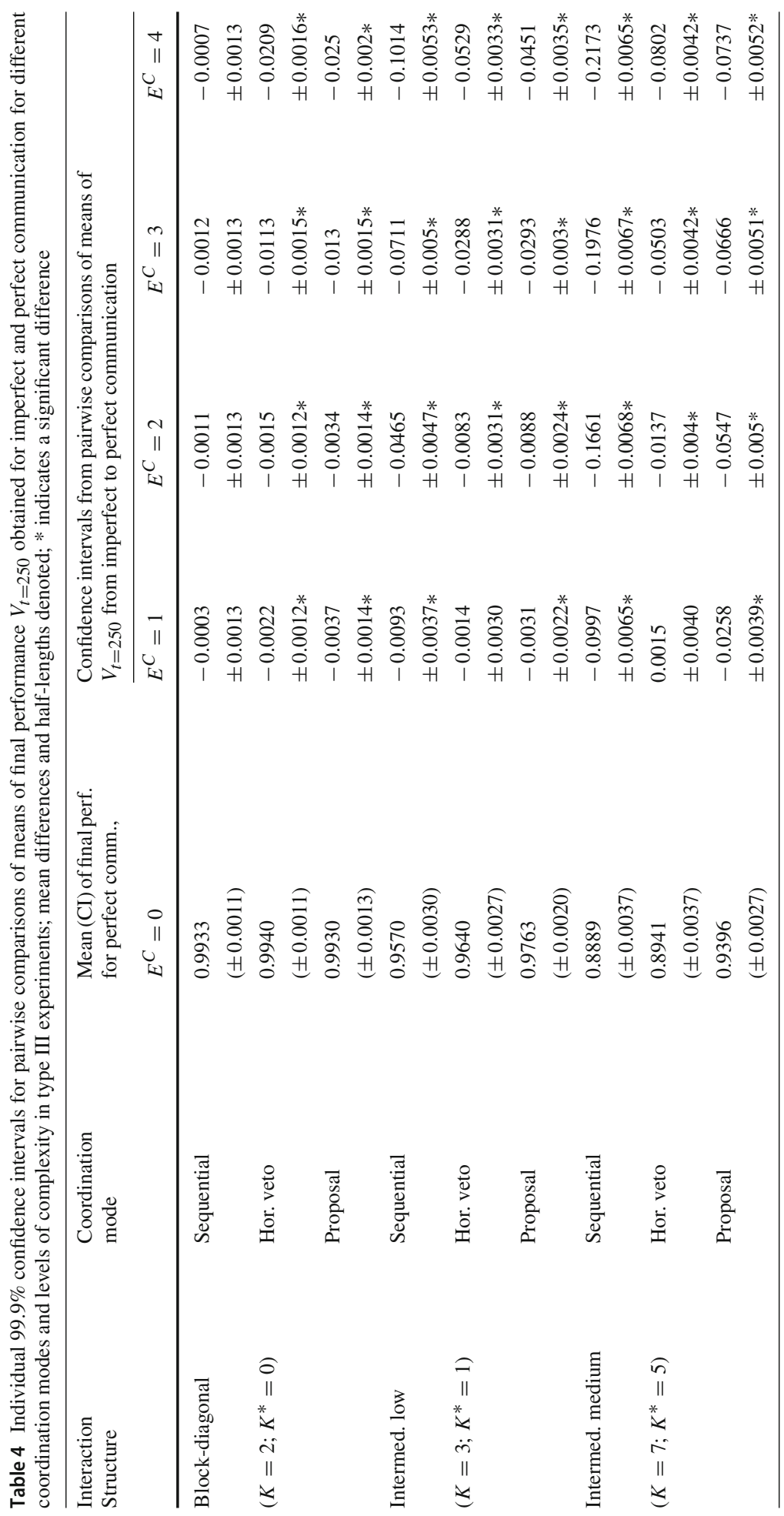

\section{照 Springer}




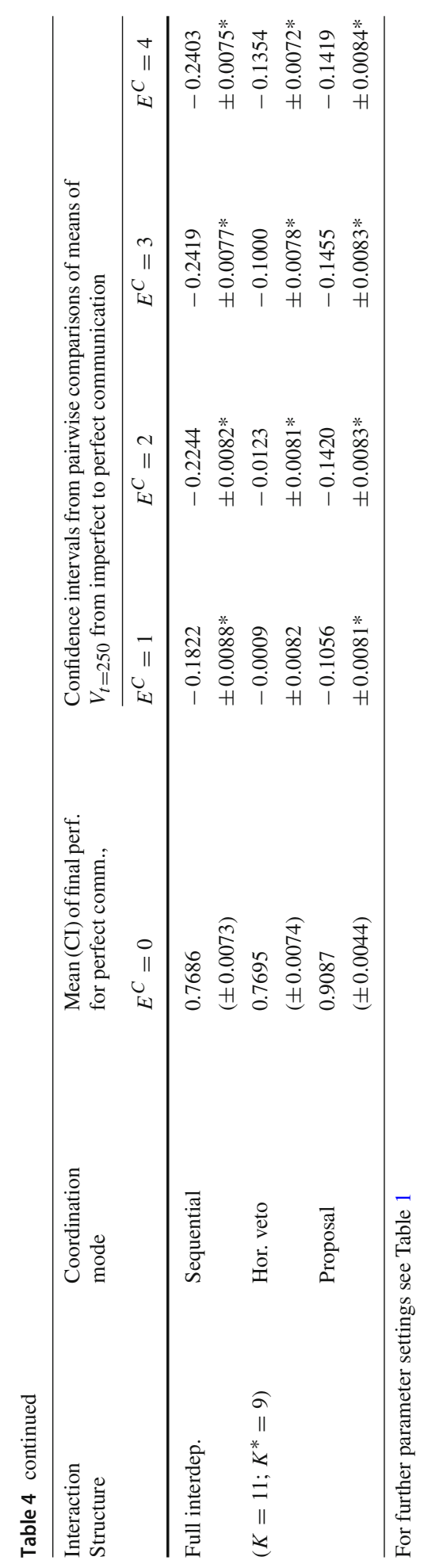




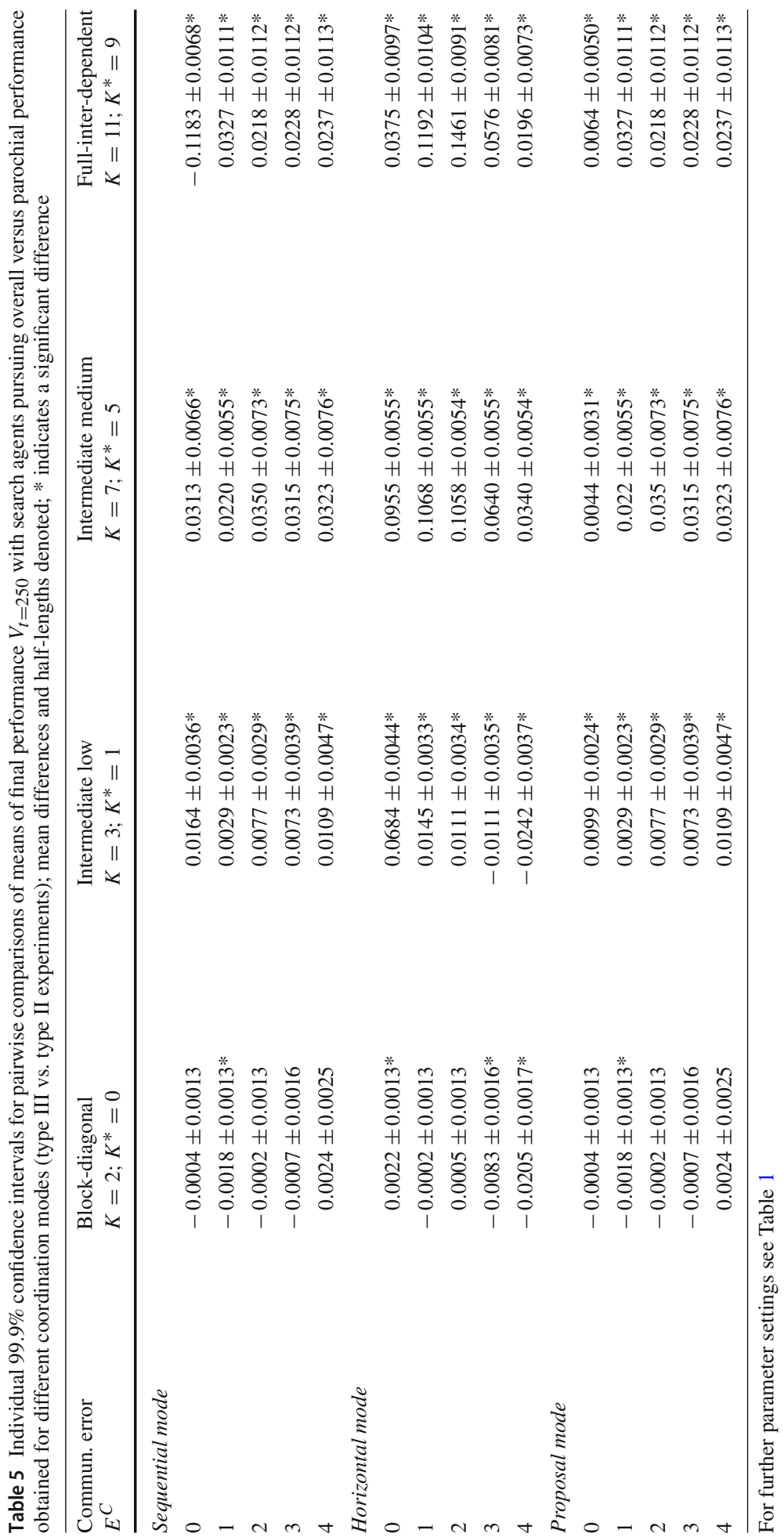




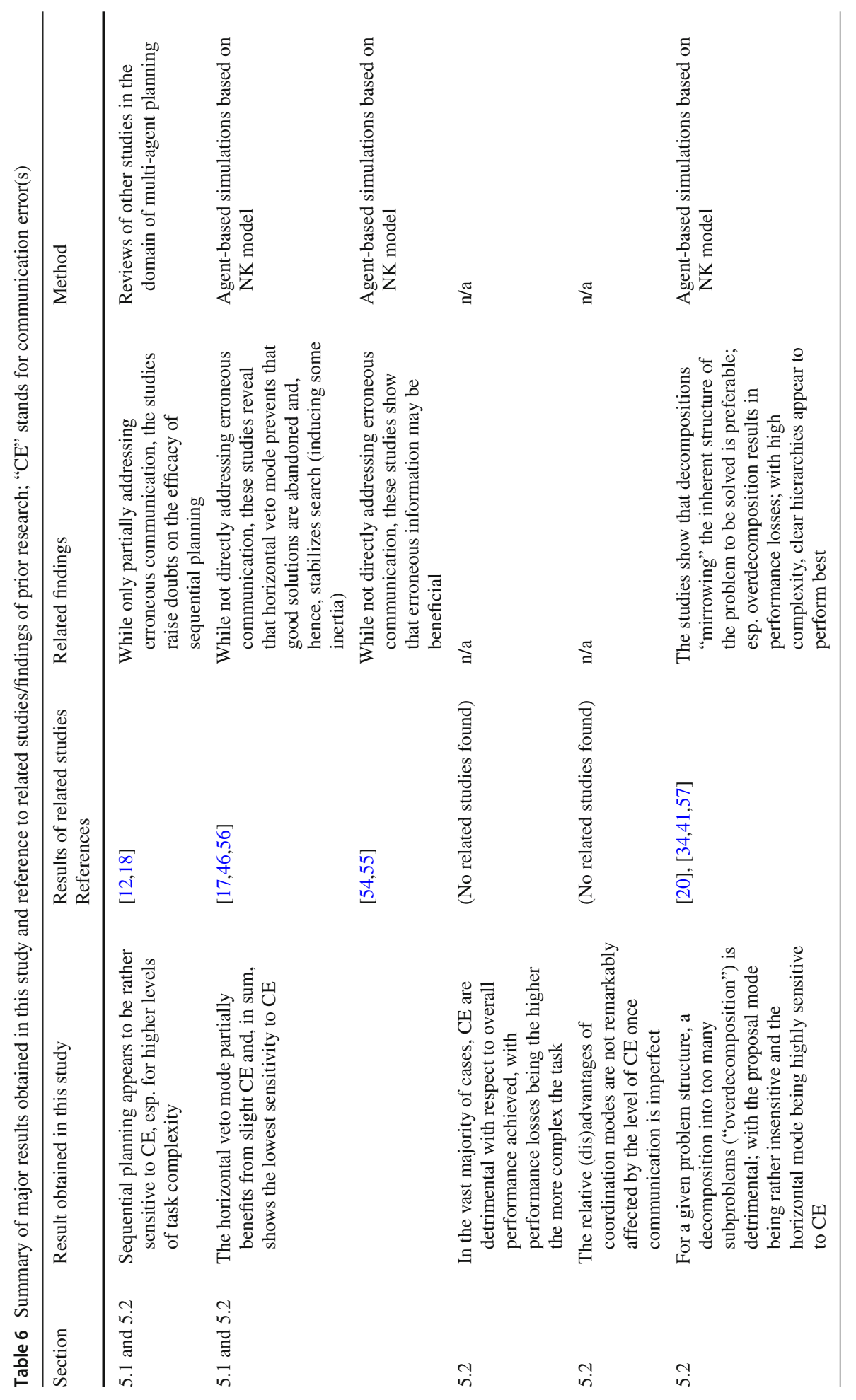




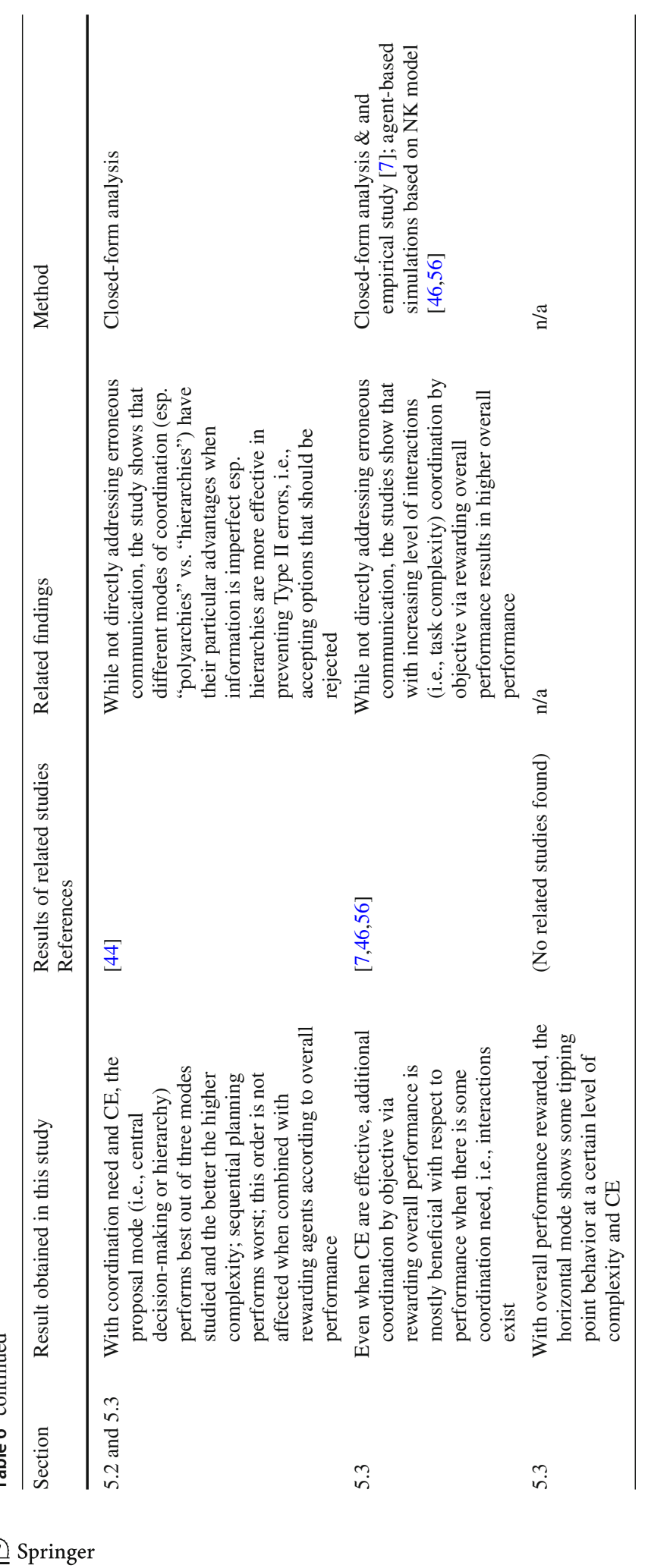




\section{References}

1. Altenberg L (1997) Section B2.7.2, NK fitness landscapes. In: Bäck T, Fogel D, Michalewicz Z (eds) Handbook of evolutionary computation. Oxford University Press, Oxford, pp B2.7:5-B2.7:10

2. Astrom KJ, Bernhardsson BM (2002) Comparison of Riemann and Lebesgue sampling for first order stochastic systems. In: Proceedings of the 41st IEEE conference on decision and control, vol 2, pp 20112016

3. Atkinson K, Bench-Capon T (2018) Taking account of the actions of others in value-based reasoning. Artif Intell 254:1-20

4. Baldwin CY, Clark KB (2000) Design rules: the power of modularity. MIT Press, Cambridge

5. Bedford DS, Malmi T, Sandelin M (2016) Management control effectiveness and strategy: an empirical analysis of packages and systems. Acc Organ Soc 51:12-28

6. Bond AH, Gasser L (1988) Chapter 1 orientation. In: Bond AH, Gasser L (eds) Readings in distributed artificial intelligence. Morgan Kauffmann, San Mateo, pp 1-56

7. Bushman RM, Indjejikian RJ, Smith A (1995) Aggregate performance measures in business unit manager compensation: the role of intrafirm interdependencies. J Acc Res 33:101-129 (supplement)

8. Cao Y, Yu W, Ren W, Chen G (2013) An overview of recent progress in the study of distributed multi-agent coordination. IEEE T Ind Inform 9:427-438

9. Carley KM (2002) Computational organizational science and organizational engineering. Simul Model Pract Theory 10:253-269

10. Cason TN, Sheremeta RM, Zhang J (2012) Communication and efficiency in competitive coordination games. Game Econ Behav 76:26-43

11. Choi S, Lee J (2014) Communication, coordination, and networks. J Eur Econ Assoc 12:223-247

12. Chaib-Draa B, Moulin B, Mandiau R, Millot P (1992) Trends in distributed artificial intelligence. Artif Intell Rev 6:35-66

13. Chow Chee W, Hwang Richard N-C, Liao W (2002) Motivating truthful upward communication of private information: an experimental study of mechanisms from theory and practice. Abacus 36:160-179

14. Daumoser C, Hirsch B, Sohn M (2018) Honesty in budgeting: a review of morality and control aspects in the budgetary slack literature. J Manage Control 29:115-159

15. Davis JP, Eisenhardt KM, Bingham CB (2007) Developing theory through simulation methods. Acad Manage Rev 32:480-499

16. Dewatripont M, Tirole J (2005) Modes of communication. J Polit Econ 113:1217-1238

17. Dosi G, Levinthal D, Marengo L (2003) Bridging contested terrain: Linking incentive-based and learning perspectives on organizational evolution. Ind Corp Change 12:413-436

18. Durfee EH (2001) Distributed problem solving and planning. In: Luck M, Marik V, Stepankova O, Trappl R (eds) Multi-agent systems and applications. Springer, Berlin, pp 118-149

19. Ellingsen T, Östling R (2010) When does communication improve coordination? Am Econ Rev 100:16951724

20. Ethiraj SK, Levinthal D (2004) Modularity and innovation in complex systems. Manage Sci 50:159-173

21. Farrell J (1988) Communication, coordination and Nash equilibrium. Econ Lett 27:209-214

22. Fioretto F, Pontelli E, Yeoh W (2018) Distributed constraint optimization problems and applications: a survey. J Artif Intell Res 61:623-698

23. Garcia E, Cao Y, Casbeer DW (2017) Periodic event-triggered synchronization of linear multi-agent systems with communication delays. IEEE Trans Autom Control 62:366-371

24. Garcia E, Cao Y, Yu H, Antsaklis P, Casbeer D (2013) Decentralised event-triggered cooperative control with limited communication. Int J Control 86:1479-1488

25. Georgeff M (1988) Communication and interaction in multi-agent planning. In: Bond AH, Gasser L (eds) Readings in distributed artificial intelligence. Morgan Kaufmann, San Mateo, pp 200-204

26. Grabner I, Moers F (2013) Management control as a system or a package? Conceptual and empirical issues. Acc Organ Soc 38:407-419

27. Kauffman SA (1993) The origins of order: self-organization and selection in evolution. Oxford University Press, Oxford

28. Kauffman SA, Levin S (1987) Towards a general theory of adaptive walks on rugged landscapes. J Theor Biol 128:11-45

29. Lambert RA (2001) Contracting theory and accounting. J Acc Econ 32:3-87

30. Larson EW, King JB (1996) The systemic distortion of information: an ongoing challenge to management. Organ Dyn 24:49-61

31. Law AM (2007) Simulation modeling and analysis, 4th edn. McGraw-Hill, New York

32. Lawrence PR, Lorsch JW (1967) Differentiation and integration in complex organizations. Admin Sci Q $12: 1-47$ 
33. Leppälä S (2013) Economic analysis of knowledge: the history of thought and the central themes. J Econ Surv 29:263-286

34. Levinthal DA, Workiewicz M (2018) When two bosses are better than one: nearly decomposable systems and organizational adaptation. Organ Sci 29:207-224

35. Li R, Emmerich MM, Eggermont J, Bovenkamp EP, Bäck T, Dijkstra J, Reiber JC (2006) Mixed-integer NK landscapes. In: Runarsson T, Beyer HG, Burke E, Merelo-Guervos J, Whitley LD, Yao X (eds) Parallel problem solving from nature-PPSN IX, LNCS, vol 4193. Springer, Berlin, pp 42-51

36. Maggi G, Rodrguez-Clare A (1995) Costly distortion of information in agency problems. RAND J Econ 26:675-689

37. Malone TW, Crowston K (1993) The interdisciplinary study of coordination. ACM Comput Surv 26:87119

38. Osband K, Reichelstein S (1985) Information-eliciting compensation schemes. J Public Econ 27:107-115

39. Parsons S, Wooldridge M (2002) Game theory and decision theory in multi-agent systems. Auton Agent Multi-Ag 5:243-254

40. Persis CD, Frasca P (2013) Robust self-triggered coordination with ternary controllers. IEEE T Autom Control 58:3024-3038

41. Querbes A, Frenken K (2018) Grounding the "mirroring hypothesis": towards a general theory of organization design in new product development. J Eng Technol Manage 47:81-95

42. Rasmußen A (2015) Reporting behavior: a literature review of experimental studies. Central Eur J Oper Res 23:283-311

43. Rivkin RW, Siggelkow N (2007) Patterned interactions in complex systems: implications for exploration. Manage Sci 53:1068-1085

44. Sah RK, Stiglitz JE (1986) The architecture of economic systems: hierarchies and polyarchies. Am Econ Rev 76:716-727

45. Siggelkow N (2002) Misperceiving interactions among complements and substitutes: organizational consequences. Manage Sci 48:900-916

46. Siggelkow N, Rivkin JW (2005) Speed and search: designing organizations for turbulence and complexity. Organ Sci 16:101-122

47. Simon HA (1955) A behavioral model of rational choice. Q J Econ 69:99-118

48. Simon HA (1959) Theories of decision-making in economics and behavioral science. Am Econ Rev 49:253-283

49. Simon HA (1991) Organizations and markets. J Econ Perspect 5:25-44

50. Stirling WC, Goodrich MA, Packard DJ (2002) Satisficing equilibria: a non-classical theory of games and decisions. Auton Agent Multi-Ag 5:305-328

51. Tonino H, Bos A, de Weerdt M, Witteveen C (2002) Plan coordination by revision in collective agent based systems. Artif Intell 142:121-145

52. von Martial F (1992) Coordinating plans of autonomous agents. In: LNAI, vol 610. Springer, Berlin. https://www.springer.com/de/book/9783540556152

53. Vorobeychik Y, Joveski Z, Yu S (2017) Does communication help people coordinate? PLOS ONE 12(2):e0170780. https://doi.org/10.1371/journal.pone.0170780

54. Wall F (2010) The (beneficial) role of informational imperfections in enhancing organisational performance. In: Li Calzi M, Milone L, Pellizzari P (eds) Progress in artificial economics. Lecture Notes in Economics and Mathematical Systems, vol 645. Springer, Berlin, pp 114-126

55. Wall F (2016) Agent-based modeling in managerial science: an illustrative survey and study. Rev Manag Sci 10:135-193

56. Wall F (2017) Learning to incentivize in different modes of coordination. Adv Complex Syst 20:1-29

57. Wall F (2018) Emergence of task formation in organizations: balancing units' competence and capacity. J Artif Soc Soc Simul 21(2):1-25. https://doi.org/10.18564/jasss.3679

58. Wall F (2018) Robustness of coordination mechanisms in distributed problem solving against erroneous communication. In: Omatu S, Rodrguez S, Villarrubia G, Faria P, Sitek P, Prieto J (eds) Distributed computing and artificial intelligence, 14th international conference. AISC, vol 620. Springer International Publishing, Cham, pp 148-156

59. Welch BL (1938) The significance of the differences between two means when the population variances are unequal. Biometrika 25:350-362

60. Wooldridge M (2009) An introduction to multiagent systems, 2nd edn. Wiley, Chichester 
Publisher's Note Springer Nature remains neutral with regard to jurisdictional claims in published maps and institutional affiliations.

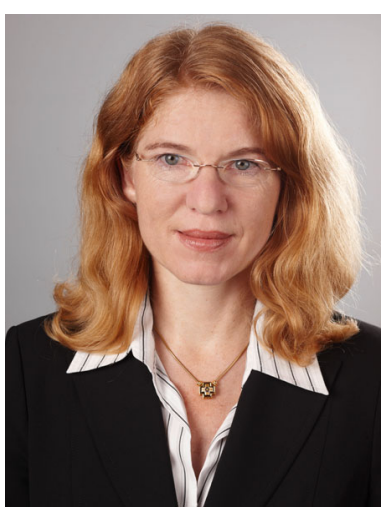

Friederike Wall is Full Professor and Head of the Department of Management Control and Strategic Management at the Alpen-AdriaUniversität Klagenfurt, Austria. Her scientific work is focused on the coordination of distributed decision-making within organizations, on management control systems and the quality of information provided by these systems. Her main research approach is defined by agentbased simulation methods and agent-based technologies. 